\title{
THE AUSLANDER-REITEN COMPONENTS SEEN AS QUASI-HEREDITARY CATEGORIES
}

\author{
MARTÍN ORTIZ-MORALES
}

\begin{abstract}
Quasi-hereditary were introduced by L. Scott Scott, CPS1, CPS2 in order to deal highest weight categories as they arise in the representation theory of semi-simple complex Lie algebras and algebraic groups, and they have been a very important tool in the study of finite-dimensional algebras. On the other hand, functor categories were introduced in representation theory by M. Auslander $[\mathrm{A}],[\mathrm{AQM}]$ and used in his proof of the first Brauer-Thrall conjecture [A2] and later on used systematically in his joint work with I. Reiten on stable equivalence [AR], [AR2] and many other applications. Recently, functor categories were used in [MVS3] to study the Auslander-Reiten components of finite-dimensional algebras. The aim of the paper is to introduce the concept of quasi-hereditary category, and we can think of the components of the Auslander-Reiten components as quasi-hereditary categories. In this way, we have applications to the functor category $\operatorname{Mod}(\mathcal{C})$, with $\mathcal{C}$ a component of the Auslander-Reiten quiver.
\end{abstract}

\section{INTRODUCTION AND BASIC CONCEPTS}

The notion of quasi-hereditary algebra was introduced by E. Cline, B. Parshall and L. Scott in their work on highest weight categories arising in representation theory of Lie algebras and algebraic groups Scott, CPS1, CPS2. Later quasihereditary algebras were amply studied by Dlab and Ringel [Dlab, DR, Rin2].

On the other hand, functor categories were introduced in representation theory by Auslander $\mathrm{A}$ and used in his proof of the first Brauer-Thrall conjecture $\mathrm{A} 2$ and later used systematically in his joint work with I. Reiten on stable equivalence and many other applications AR, AR2. Recently, functor categories were employed by Martínez-Villa and Solberg to study the Auslander-Reiten components of finitedimensional algebras [MVS3] and to develop tilting theory in arbitrary functor categories MVO1, MVO2].

In [MVS3], by using the concepts and the results on Koszul and Artin-Schelter regular categories from MVS1, MVS2, results analogous to those presented by Auslander in [A, Theorems 3.12 and 3.13] were shown. There Auslander characterizes when the category of all additive contravariant functors from the category of finitely generated modules over a finite-dimensional algebra $\Lambda$ to abelian groups is left (or right) Noetherian (as defined in $[\mathbf{M}]$ ). This happens if and only if the algebra $\Lambda$ is of finite representation type (that is, has only a finite number of non-isomorphic indecomposable finitely generated modules). However, instead of considering the functor category $\operatorname{Mod}(\bmod \Lambda)$, they consider the category of all additive contravariant graded functors from the associated graded category of the category of finitely

Date: October 1, 2015.

1991 Mathematics Subject Classification. 2000]Primary 05C38, 15A15; Secondary 05A15, $15 \mathrm{~A} 18$.

Key words and phrases. Quasi-hereditary algebras, Functor categories.

The author thanks PROMEP/103.5/13/6535 for giving him financial support for the development of this project.

This paper is in final form, and no version of it will be submitted for publication elsewhere. 
generated $\Lambda$-modules to graded vector spaces. The associated graded category of the finitely generated left $\Lambda$-modules with respect to the radical of the category is a disjoint union of all the associated graded categories of the additive closures of all the components $\mathcal{K}$ in the Auslander-Reiten quiver. Therefore, they reduced the problem to consider one component $\mathcal{K}$ at the time and the associated graded category $\mathcal{A}_{\text {gr }}(\mathcal{K})$ of that component $\mathcal{K}$. They showed that for regular components $\mathcal{K}$ the category of graded functors from $\mathcal{A}_{\text {gr }}(\mathcal{K})$ to graded vector spaces is left Noetherian if and only if the sections of $\mathcal{K}$ are infinite Dynkin diagrams $A_{\infty}, A_{\infty}^{\infty}, D_{\infty}$ or extended Dynkin diagrams.

In the same spirit as in the above-mentioned results, in this paper we consider applications to functor categories: categories formed by functors defined on a subcategory $\mathcal{C}$ of the category of modules over a finite-dimensional algebra which take values in the category of abelian groups. We generalize the theory of quasihereditary algebras from modules to functor categories, having in mind as one of the main motivations to apply the developed results to study the Auslander-Reiten componentes of finite-dimensional algebras seen as quasi-hereditary categories.

This paper consists of four sections. In the first section, we fix the notation and recall some notions from functor categories that will be used throughout the paper. In the second section, we generalize some results about quasi-hereditary algebras from modules to functor categories, generalizing the concept of sequence of standard modules from modules to sequence of standard subcategories $\Delta$ of $\mathcal{C}$. In addition we give some characterizations of the subcategory $\mathcal{F}(\Delta)$ consisting of the functors which have a $\Delta$-filtration, starting with a Hom-finite Krull-Schmidt category we obtain general results and after we add some other conditions like dualizing and Noetherian, in order to obtain similar results for the case of finitedimensional algebras. In the third section, we show that the category $\mathcal{F}(\Delta)$ is functorially finite if $\mathcal{C}$ is dualizing, Krull-Schmidt, Noetherian and quasi-hereditary with respect to a finite filtration (see [Theorem 1, Rin2]). The fourth section is dedicated to the examples in which we exhibit some filtrations for the different Auslander-Reiten components to consider them and as quasi-hereditary categories. We also obtain ad hoc a tilting category for $\mathbb{Z} A_{\infty}$. Finally, we show that tensor product of quasi-hereditary categories is again a quasi-hereditary category.

1.1. The category $\operatorname{Mod}(\mathcal{C})$. Throughout this section $\mathcal{C}$ will be an arbitrary skeletally small preadditive category, and $\operatorname{Mod}(\mathcal{C})$ will denote the category of contravariant functors from $\mathcal{C}$ to the category of abelian groups. According to Mitchell $[\mathrm{M}$, we can think of $\mathcal{C}$ as a ring "with several objects" and $\operatorname{Mod}(\mathcal{C})$ as a category of $\mathcal{C}$-modules. The aim of the paper is to show that the notions of quasi-hereditary algebras can be extended to $\operatorname{Mod}(\mathcal{C})$, obtaining as a reult generalizations of some results on quasi-hereditary algebras. In order to obtain generalizations of some theorems for quasi-hereditary algebras on finite-dimensional algebras, we need to add restrictions to our category $\mathcal{C}$, such as Krull-Schmidt, Hom-finite, dualizing, etc.. To fix the notation, we refer to known results on functors and categories that we use throughout the paper, making reference to the papers by Auslander and Reiten $\mathrm{A}$, AQM, $\mathrm{AR}$.

$\operatorname{Mod}(\mathcal{C})$ is then an abelian category with arbitrary sums and products; in fact it has arbitrary limits and colimits, and the filtered limits are exact (Ab5 in Grothendieck terminology). Furthermore, $\operatorname{Mod}(\mathcal{C})$ has enough projective and injective objects. For any object $C \in \mathcal{C}$, the representable functor $\mathcal{C}(, C)$ is projective, the arbitrary sums of representable functors are projective, and any object $M \in \operatorname{Mod}(\mathcal{C})$ is covered by an epimorphism $\coprod_{i} \mathcal{C}\left(, C_{i}\right) \rightarrow M \rightarrow 0$. We say that an object $M$ in $\operatorname{Mod}(C)$ is finitely generated if there exists an epimorphism $\coprod_{i \in I} \mathcal{C}\left(, C_{i}\right) \rightarrow M \rightarrow 0$, with $I$ a finite set. 
An object $P$ in $\operatorname{Mod}(\mathcal{C})$ is projective (finitely generated projective) if and only if $P$ is a summand of $\coprod_{i \in I} \mathcal{C}\left(, C_{i}\right)$ for a (finite) family $\left\{C_{i}\right\}_{i \in I}$ of objects in $\mathcal{C}$. The subcategory $\mathfrak{p}(\mathcal{C})$ of $\operatorname{Mod}(\mathcal{C})$, of all finitely generated projective objects, is a skeletally small additive category in which idempotents split; morever, the functor $P: \mathcal{C} \rightarrow \mathfrak{p}(\mathcal{C}), P(C)=\mathcal{C}(, C)$ is fully faithful and induces by restriction res : $\operatorname{Mod}(\mathfrak{p}(\mathcal{C})) \rightarrow \operatorname{Mod}(\mathcal{C})$ an equivalence of categories. For this reason, we may assume that our categories are skeletally small additive categories in which idempotents split; they are then called annuli varieties in $[\mathrm{A}$.

Definition 1.1. AQM Given a preadditive skeletally small category $\mathcal{C}$, we say $\mathcal{C}$ has pseudokernels, if given a map $f: C_{1} \rightarrow C_{0}$ there exists a map $g: C_{2} \rightarrow C_{1}$ such that the sequence $\mathcal{C}\left(, C_{2}\right) \stackrel{(, g)}{\rightarrow} \mathcal{C}\left(, C_{1}\right) \stackrel{(, f)}{\rightarrow} \mathcal{C}\left(, C_{0}\right)$ is exact.

A functor $M$ is finitely presented if there exists an exact sequence $\mathcal{C}\left(, C_{1}\right) \rightarrow$ $\mathcal{C}\left(, C_{0}\right) \rightarrow M \rightarrow 0$. We denote by $\bmod (\mathcal{C})$ the full subcategory of $\operatorname{Mod}(\mathcal{C})$ consisting of finitely presented functors. It was proved in $\mathrm{AQM}$ that $\bmod (\mathcal{C})$ is abelian if and only if $\mathcal{C}$ has pseudokernels.

We will indistinctly say that $M$ is an object of $\operatorname{Mod}(\mathcal{C})$ or that $M$ is a $\mathcal{C}$-module. A representable functor $\mathcal{C}(, C)$ will sometimes be denoted by $(, C)$.

1.2. Krull-Schmidt and Dualizing Categories. We start by giving some definitions from [AR].

Definition 1.2. Let $R$ be a commutative Artin ring. A $R$-category $\mathcal{C}$, is a preadditive category such that $\mathcal{C}\left(C_{1}, C_{2}\right)$ is a $R$-module and, the composition is $R$-bilinear. Under these conditions $\operatorname{Mod}(\mathcal{C})$ is a $R$-category which we identify with the category of functors $\left(\mathcal{C}^{o p}, \operatorname{Mod}(R)\right)$.

$A R$-category $\mathcal{C}$ is Hom-finite if for each pair of objects $C_{1}, C_{2}$ in $\mathcal{C}$ the $R$-module $\mathcal{C}\left(C_{1}, C_{2}\right)$ is finitely generated. We denote by $\left(\mathcal{C}^{o p}, \bmod (R)\right)$ the full subcategory of $\left(\mathcal{C}^{o p}, \operatorname{Mod}(R)\right)$ consisting of the $\mathcal{C}$-modules such that for every $C$ in $\mathcal{C}$ the $R$-module $M(C)$ is finitely generated. The category $\left(\mathcal{C}^{o p}, \bmod (R)\right)$ is abelian, and the inclusion $\left(\mathcal{C}^{o p}, \bmod (R)\right) \rightarrow\left(\mathcal{C}^{o p}, \operatorname{Mod}(R)\right)$ is exact.

The category $\bmod (C)$ is a full subcategory of $\left(\mathcal{C}^{o p}, \bmod (R)\right)$. The functors $D$ : $\left(\mathcal{C}^{o p}, \bmod (R)\right) \rightarrow(\mathcal{C}, \bmod (R))$ and $D:(\mathcal{C}, \bmod (R)) \rightarrow\left(\mathcal{C}^{o p}, \bmod (R)\right)$ are defined as follows: for any $C$ in $\mathcal{C}, D(M)(C)=\operatorname{Hom}_{R}(M(C), I(R / r)$ ), with $r$ the Jacobson radical of $R$, and $I(R / r)$ is the injective envelope of $R / r$. The functor $D$ defines a duality between $(\mathcal{C}, \bmod (R))$ and $\left(\mathcal{C}^{o p}, \bmod (R)\right)$. If $\mathcal{C}$ is a Hom-finite $R$-category and $M$ is in $\bmod (\mathcal{C})$, then $M(C)$ is a finitely generated $R$-module and is therefore in $\bmod (R)$.

Definition 1.3. A Hom-finite $R$-category $\mathcal{C}$ is dualizing, if the functor $D$ : $\left(\mathcal{C}^{o p}, \bmod (R)\right) \rightarrow(\mathcal{C}, \bmod (R))$ induces a duality between the categories $\bmod (\mathcal{C})$ and $\bmod \left(\mathcal{C}^{o p}\right)$.

It is clear from the definition that for dualizing categories $\bmod (\mathcal{C})$ has enough injectives. Morever, $\mathcal{C}$ has pseudokerneles, and therefore $\bmod (\mathcal{C})$ is an abelian category by Theorem 2.4 in $\mathrm{AR}$.

To finish, we recall the following definition.

Definition 1.4. An additive category $\mathcal{C}$ is $\mathbf{K r u l l - S c h m i d t}$, if every object in $\mathcal{C}$ decomposes in a finite sum of objects whose endomorphism ring is local.

Assume $\mathcal{C}$ is Krull-Schmidt, then for all $C \in \mathcal{C}$ the ring of endomorphisms $\operatorname{End}(C)$ is semiperfect. In other, every $\operatorname{End}(C)$-module finitely generated has a projective cover and therefore every finitely presented $\mathcal{C}$-module has a minimal projective presentation by Theorem 1 and Theorem 2 in [MVO2]. 
1.3. Change of categories. According to $[\mathrm{A}]$, there exists a unique up to isomorphism functor $-\otimes_{\mathcal{C}}-: \operatorname{Mod}\left(\mathcal{C}^{o p}\right) \times \operatorname{Mod}(\mathcal{C}) \rightarrow \mathbf{A b}$, called the tensor product, with the following properties:

(a) (i) For each $\mathcal{C}$-module $N$, the functor $\otimes_{\mathcal{C}} N: \operatorname{Mod}\left(\mathcal{C}^{o p}\right) \rightarrow \mathbf{A b}$ given by $\left(\otimes_{\mathcal{C}} N\right)(M)=M \otimes_{\mathcal{C}} N$ is right exact.

(ii) For each $\mathcal{C}^{o p}$-module $M$, the functor $M \otimes_{\mathcal{C}}: \operatorname{Mod}(\mathcal{C}) \rightarrow \mathbf{A b}$ given by $\left(M \otimes_{\mathcal{C}}\right)(N)=M \otimes_{\mathcal{C}} N$ is right exact.

(b) The functors $M \otimes_{\mathcal{C}}-$ and $-\otimes_{\mathcal{C}} N$ preserve arbitrary sums.

(c) For each object $C$ in $\mathcal{C} M \otimes_{\mathcal{C}}(, C)=M(C)$ and $(C, \quad) \otimes_{\mathcal{C}} N=N(C)$.

Given a full subcategory $\mathcal{C}^{\prime}$ of $\mathcal{C}$, the restriction res $: \operatorname{Mod}(\mathcal{C}) \rightarrow \operatorname{Mod}\left(\mathcal{C}^{\prime}\right)$ has a right adjoint, also called the tensor product, and denoted by $\mathcal{C} \otimes_{\mathcal{C}^{\prime}}: \operatorname{Mod}\left(\mathcal{C}^{\prime}\right) \rightarrow$ $\operatorname{Mod}(\mathcal{C})$. This functor is defined by $\left(\mathcal{C} \otimes_{\mathcal{C}^{\prime}} M\right)(C)=\left.(C, \quad)\right|_{\mathcal{C}^{\prime}} \otimes_{\mathcal{C}^{\prime}} M$, for any $M$ in $\operatorname{Mod}\left(\mathcal{C}^{\prime}\right)$ and $C$ in $\mathcal{C}$. The following proposition is proved in [A Prop. 3.1].

Proposition 1.5. Let $\mathcal{C}^{\prime}$ be a full subcategory of $\mathcal{C}$. The functor $\mathcal{C} \otimes_{\mathcal{C}^{\prime}}: \operatorname{Mod}\left(\mathcal{C}^{\prime}\right) \rightarrow$ $\operatorname{Mod}(\mathcal{C})$ satisfies the following conditions:

(a) $\mathcal{C} \otimes_{\mathcal{C}^{\prime}}$ is right exact and preserves arbitrary sums.

(b) The composition $\operatorname{Mod}\left(\mathcal{C}^{\prime}\right) \stackrel{\mathcal{C} \otimes_{\mathcal{C}^{\prime}}}{\longrightarrow} \operatorname{Mod}(\mathcal{C}) \stackrel{\text { res }}{\longrightarrow} \operatorname{Mod}\left(C^{\prime}\right)$ is the identity in $\operatorname{Mod}\left(\mathcal{C}^{\prime}\right)$.

(c) For each object $C^{\prime}$ in $\mathcal{C}^{\prime}, \mathcal{C} \otimes \mathcal{C}^{\prime} \mathcal{C}^{\prime}\left(, C^{\prime}\right)=\mathcal{C}\left(, C^{\prime}\right)$.

(d) $\mathcal{C} \otimes_{\mathcal{C}^{\prime}}$ is fully faithful.

(e) $\mathcal{C} \otimes_{\mathcal{C}^{\prime}}$ preserves projective objects.

The functor $M$ in $\operatorname{Mod}(\mathcal{C})$ is called projectively presented over $\mathcal{C}^{\prime}$, if there exists an exact sequence $\coprod_{i \in I} \mathcal{C}\left(\quad, C_{i}^{\prime}\right) \rightarrow \coprod_{j \in J} \mathcal{C}\left(, C_{j}^{\prime}\right) \rightarrow M \rightarrow 0$, with $C_{i}^{\prime}, C_{j}^{\prime} \in \mathcal{C}^{\prime}$. The category $\mathcal{C} \otimes_{\mathcal{C}^{\prime}} \operatorname{Mod}\left(\mathcal{C}^{\prime}\right)$ is the subcategory of $\operatorname{Mod}(\mathcal{C})$ with objects that are the functors projectively presented over $\mathcal{C}^{\prime}$. The functors res and $\mathcal{C} \otimes \mathcal{C}^{\prime}$ induce an equivalence between $\operatorname{Mod}\left(\mathcal{C}^{\prime}\right)$ and $\mathcal{C} \otimes_{\mathcal{C}^{\prime}} \operatorname{Mod}\left(\mathcal{C}^{\prime}\right)$.

1.4. The radical of a category. The notion of the Jacobson radical of a category was introduced in $[\mathrm{M}]$ and $[\mathrm{A}]$, it is defined in the following way:

Definition 1.6. The Jacobson radical of $\mathcal{C}, \operatorname{rad}_{\mathcal{C}}($,$) is a subbifunctor of \operatorname{Hom}_{\mathcal{C}}($, defined in objects as $\operatorname{rad}_{\mathcal{C}}(X, Y)=\left\{f \in \operatorname{Hom}_{\mathcal{C}}(X, Y) \mid\right.$ for any map $g: Y \rightarrow X$, $1-g f$ is invertible $\}$.

If $M$ is a $\mathcal{C}$-module, then we denote by $\operatorname{rad} M$ the intersection of all maximal subfunctors of $M$.

Proposition 1.7. $[\mathrm{A}],[\mathrm{BR}],[\mathrm{M}]$ Let $\mathcal{C}$ be an additive category and $\operatorname{rad}_{\mathcal{C}}($,$) the$ Jacobson radical of $\mathcal{C}$. We then have the following:

(a) For every object $C$ in $\mathcal{C}, \operatorname{rad}_{\mathcal{C}}(C, C)$ is just the Jacobson radical of $\operatorname{End}_{\mathcal{C}}(C)$.

(b) If $C$ and $C^{\prime}$ are indecomposable objects in $\mathcal{C}$, then $\operatorname{rad}_{\mathcal{C}}\left(C, C^{\prime}\right)$ consists of all non isomorphisms from $C$ to $C^{\prime}$.

(c) For every object $C$ in $\mathcal{C}, \operatorname{rad}_{\mathcal{C}}(C, \quad)=\operatorname{rad} \mathcal{C}(C, \quad)$ and $\operatorname{rad}_{\mathcal{C}}(, C)=$ $\operatorname{rad} \mathcal{C}(, C)$.

(d) For every pair of objects $C$ and $C^{\prime}$ in $\mathcal{C}, \operatorname{rad}_{\mathcal{C}}\left(C^{\prime}, \quad\right)(C)=\operatorname{rad}_{\mathcal{C}}(, C)\left(C^{\prime}\right)$.

Definition 1.8. By an ideal of the additive category $\mathcal{C}$, we understand a subbifunctor of $\operatorname{Hom}_{\mathcal{C}}($,$) .$

Given two ideals $I_{1}$ and $I_{2}$ of $\mathcal{C}$, we define $I_{1} I_{2}$ as follows: $f \in I_{1} I_{2}\left(C_{1}, C_{3}\right)$, if and only if $f$ is a finite sum of morphisms $C_{1} \stackrel{h}{\rightarrow} C_{2} \stackrel{g}{\rightarrow} C_{3}$, with $h \in I_{1}\left(C_{1}, C_{2}\right)$ and $g \in I_{2}\left(C_{2}, C_{3}\right)$. 


\section{Quasi-hereditary Categories}

In DR] quasi-hereditary rings are introduced in terms of filtrations of idempotent ideals; meanwhile in [Dlab, A.2.6 ] quasi-hereditary algebras are defined in terms of filtrations into ideales determined by traces of certain projective modules.

In this work we assume that $\mathcal{C}$ is a Hom-finite $K$-category, with an algebraically closed field $K$.

Here we introduce the concept of quasi-hereditary category by bringing the concepts mentioned above, bearing in mind generalize some classical results on quasihereditary algebras. To achieve this, we begin studying certain types of idempotent ideals in a $K$-category $\mathcal{C}$. We then introduce the concept of heredity ideal (see $[\mathrm{DR}]$ ), so we can define quasi-hereditary categories through a filtration of the bifunctor $\mathcal{C}(-, ?)$ into ideals, which corresponds to a filtration of the category $\mathcal{C}$ into subcategories.

In the main theorem of this section, we give conditions on the filtration of a category in subcategories to make this a quasi-hereditary category.

2.1. The ideal $I_{\mathcal{B}}(-$, ?). We start this section by studying an important ideal that will be very important in the study of quasi-hereditary categories.

Let $\mathcal{B}$ be a full subcategory of $\mathcal{C}$. We denote by $I_{\mathcal{B}}\left(C, C^{\prime}\right)$ the subgroup of $\mathcal{C}\left(C, C^{\prime}\right)$ consisting of morphisms which factor through some object of $\mathcal{B}$. Let $E \in \mathcal{B}$ and $k_{E}=\operatorname{dim}_{K} \mathcal{C}(E, X)$. If $\left\{f_{1}, \ldots, f_{k_{E}}\right\}$ is a $K$-basis for $\mathcal{C}(E, X)$, then we can define a map for all $C \in \mathcal{C}$ :

$$
\begin{aligned}
\varphi_{C}^{E} \quad: \quad \mathcal{C}(C, E)^{k_{E}} & \rightarrow \mathcal{C}(C, X) \\
\left(g_{1}, \ldots, g_{k_{E}}\right) & \mapsto \sum_{i=1}^{k_{E}} f_{i} g_{i}
\end{aligned}
$$

We can readily ascertain that $\varphi^{E}: \mathcal{C}(-, E)^{k_{E}} \rightarrow \mathcal{C}(-, X)$ is a morphism of $\mathcal{C}$ modules. Therefore, we have an induced morphism $\left(\varphi^{E}\right)_{E \in \mathcal{B}}: \coprod_{E \in \mathcal{B}} \mathcal{C}(-, E)^{k_{E}} \rightarrow$ $\mathcal{C}(-, X)$.

In the next lemma we study the relationship between the ideal $I_{\mathcal{B}}(-$, ?) and the trace $\operatorname{Tr}_{\{\mathcal{C}(-, E)\}_{E \in \mathcal{B}}} \mathcal{C}(-, X)$ of the family of projective $\mathcal{C}$-modules $\left\{\mathcal{C}(-, E)_{E \in \mathcal{B}}\right\}$ in $\mathcal{C}(-, X)$.

Lemma 2.1. Let $\left(\varphi^{E}\right)_{E \in \mathcal{B}}: \coprod_{E \in \mathcal{B}} \mathcal{C}(-, E)^{k_{E}} \rightarrow \mathcal{C}(-, X)$ the morphism defined as above. Then $\operatorname{Im}\left(\left(\varphi^{E}\right)_{E \in \mathcal{B}}\right)=\operatorname{Tr}_{\{\mathcal{C}(-, E)\}_{E \in \mathcal{B}}} \mathcal{C}(-, X)=I_{\mathcal{B}}(-, X)$.

Proof. a) For all $E \in \mathcal{B}$ we have $\operatorname{Im}\left(\varphi^{E}\right)=\sum_{\hat{f} \in \mathcal{C}(E, X)} \operatorname{Im}(\mathcal{C}(-, \hat{f}))$. Indeed let $\left\{f_{1}, \ldots f_{k_{E}}\right\}$ be a $K$-basis for $\mathcal{C}(E, X)$; we then have

$$
\operatorname{Im}\left(\varphi_{C}^{E}\right)=\left\{\sum_{i=1}^{k_{E}} f_{i} g_{i} \mid g_{i} \in \mathcal{C}(C, E)\right\}
$$

On the other hand, we have

$$
\begin{aligned}
\sum_{\hat{f} \in \mathcal{C}(E, X)} \operatorname{Im}\left(\mathcal{C}(-, \hat{f})_{C}\right) & =\sum_{\hat{f} \in \mathcal{C}(E, X)} \operatorname{Im}(\mathcal{C}(C, \hat{f})) \\
& =\left\{\sum_{s=1}^{m} \hat{f}_{s} g_{s} \mid \hat{f}_{s} \in \mathcal{C}(E, X), g_{s} \in \mathcal{C}(C, E), m \geq 0\right\}
\end{aligned}
$$


It is clear that $\operatorname{Im}\left(\varphi^{E}\right) \subset \sum_{\hat{f} \in \mathcal{C}(E, X)} \operatorname{Im}(\mathcal{C}(-, \hat{f}))$. To prove the other inclusion, let $\hat{f}_{s}=\sum_{i=1}^{k_{E}} c_{i s} f_{i}, c_{i s} \in K$, a map in $\mathcal{C}(E, S)$. Then, it follows that

$$
\sum_{s=1}^{m} \hat{f}_{s} g_{s}=\sum_{s=1}^{m}\left(\sum_{i=1}^{k_{E}} c_{i s} f_{i}\right) g_{s}=\sum_{i=1}^{k_{E}} f_{i}\left(\sum_{s=1}^{m} c_{i s} g_{s}\right)
$$

Thus, $\sum_{\hat{f} \in \mathcal{C}(E, X)} \operatorname{Im}(\mathcal{C}(-, \hat{f})) \subset \operatorname{Im}\left(\varphi^{E}\right)$.

b) For all $E \in \mathcal{B}$, we have $\operatorname{Im}\left(\varphi^{E}\right)=\operatorname{Tr}_{(-, E)} \mathcal{C}(-, X)$. By Yoneda's lemma it follows that

$$
\operatorname{Tr}_{(-, E)} \mathcal{C}(-, X)=\sum_{\psi \in(\mathcal{C}(-, E), \mathcal{C}(-, X))} \operatorname{Im}(\psi)=\sum_{\hat{f} \in \mathcal{C}(E, X)} \operatorname{Im}(\mathcal{C}(-, \hat{f}))
$$

c) Finally, observe that $f \in \operatorname{Im}\left(\left(\varphi^{E}\right)_{E \in \mathcal{B}}\right)(C)$ if and only if $f \in I_{\mathcal{B}}(C, X)$.

2.2. Heredity ideals and quasi-hereditary categories. In order to introduce the concept of quasi-hereditary category we first introduce the concept of heredity ideal $\mathrm{DR}$.

Definition 2.2. A two-sided ideal $I$ in $\mathcal{C}$ is called heredity if the following holds:

(i) $I^{2}=I$, i.e, $I$ is an idempotent ideal;

(ii) $\operatorname{Irad} \mathcal{C}(-, ?) I=0$;

(iii) $I(-, X)$ is a finitely generated projective $\mathcal{C}$-module for all $X \in \mathcal{C}$.

Let $\mathcal{B} \subset \mathcal{C}$ a full subcategory; it is clear that $I_{\mathcal{B}}^{2}=I_{\mathcal{B}}$. Then, we want to know when the ideal $I_{\mathcal{B}}$ is heredity. The following lemma says when it holds.

Lemma 2.3. Let $\mathcal{B}$ be a full subcategory of $\mathcal{C}$. Then, the ideal $I_{\mathcal{B}}$ is heredity if and only if the following holds:

(i) $\operatorname{rad} \mathcal{C}\left(E_{1}, E_{2}\right)=0$ for all pairs of non-isomorphic objects $E_{1}, E_{2} \in \mathcal{B}$;

(ii) $I_{\mathcal{B}}(-, X)$ is a finite direct sum of projective $\mathcal{C}$-modules of the form $\mathcal{C}(-, E)$, $E \in \mathcal{B}$, for all $X \in \mathcal{C}$.

Proof. (i) Assume that $I_{\mathcal{B}} \operatorname{rad} \mathcal{C}\left(-\right.$, ?) $I_{\mathcal{B}}=0$, and let $E_{1}, E_{2} \in \mathcal{B}$ a pair of nonisomorphic objects. Assume that $t \in \operatorname{rad} \mathcal{C}\left(E_{1}, E_{2}\right)$. We then have

$$
t=1_{E_{2}} t 1_{E_{1}} \in I_{\mathcal{B}}\left(E_{1}, E_{1}\right) \operatorname{rad} \mathcal{C}\left(E_{1}, E_{2}\right) I_{\mathcal{B}}\left(E_{2}, E_{2}\right)=0 .
$$

Thus, $\operatorname{rad} \mathcal{C}\left(E_{1}, E_{2}\right)=0$. Conversely, assume that $\operatorname{rad} \mathcal{C}\left(E_{1}, E_{2}\right)=0$ for all pairs of non-isomorphic objects $E_{1}, E_{2} \in \mathcal{B}$. Let $f \in I_{\mathcal{B}}(X, Y) \operatorname{rad} \mathcal{C}(Y, Z) I_{\mathcal{B}}(Z, W)$ which has the form $f=r s t$ with $r \in I_{\mathcal{B}}(X, Y), t \in \operatorname{rad} \mathcal{C}(Y, Z)$ and $s \in I_{\mathcal{B}}(Z, W)$. Therefore, we can write these maps as $r: X \rightarrow \coprod B_{i} \rightarrow Y$ and $s: Z \rightarrow \coprod B_{j} \rightarrow W$, where the terms in the middle are finite sums of indecomposable objects in $\mathcal{B}$. It follows that the induced maps $B_{i} \rightarrow Y \stackrel{t}{\rightarrow} Z \rightarrow B_{j}$ are all in $\operatorname{rad} \mathcal{C}\left(B_{i}, B_{j}\right)=0$. Finally, $f=0$.

(ii) By Lemma 2.1, there exists an epimorphism $\coprod_{i=1}^{n} \mathcal{C}\left(-, E_{i}\right) \rightarrow I_{\mathcal{B}}(-, X) \rightarrow 0$, $E_{i} \in \mathcal{B}$. Thus, $I_{\mathcal{B}}(-, X)$ is a projective finitely generated $\mathcal{C}$-module if and only if $I_{\mathcal{B}}(-, X)$ is a finite direct sum of projective $\mathcal{C}$-modules of the form $\mathcal{C}(-, E), E \in \mathcal{B}$.

In order to generalize the definition given in $[\mathrm{DR}]$, we have the following.

Definition 2.4. Let $K$ be an algebraically closed field. A K-category is called quasi-hereditary if for all $C \in \mathcal{C}$ there exists a chain of two-sided ideals

$$
0=I_{0} \subset I_{1} \subset I_{2} \cdots I_{i-1} \subset I_{i} \subset \cdots \subset \mathcal{C}(-, ?),
$$

which is exhaustive (i.e., $\cup_{i>0} I_{i}=\mathcal{C}\left(-\right.$, ?)), and $I_{i} / I_{i-1}$ is heredity in the category $\mathcal{C} / I_{i-1}$. Such a chain is called a heredity chain. 
Assume we have a filtration $\left\{\mathcal{B}_{i}\right\}_{i \geq 0}$ of $\mathcal{C}$ into subcategories

$$
\{0\}=\mathcal{B}_{0} \subset \mathcal{B}_{1} \subset \cdots \subset \mathcal{C},
$$

which is exhaustive (i.e., $\cup_{i \geq 0} \mathcal{B}_{i}=\mathcal{C}$ ). Then, we have an exhaustive chain of two sided ideals

$$
0=I_{\mathcal{B}_{0}} \subset I_{\mathcal{B}_{1}} \subset \cdots \subset I_{\mathcal{B}_{i-1}} \subset I_{\mathcal{B}_{i}} \subset \cdots \subset \mathcal{C}(-, ?) .
$$

In relation to the above definition, we want to know when (11) is a heredity chain. It is clear that $I_{\mathcal{B}_{i}} / I_{\mathcal{B}_{i-1}}$ is an idempotent ideal in the category $\mathcal{C} / I_{i-1}$, because $I_{\mathcal{B}_{i}}$ and $I_{\mathcal{B}_{i-1}}$ are idempotent in $\mathcal{C}$ and

$$
\left(\frac{I_{\mathcal{B}_{i}}}{I_{\mathcal{B}_{i-1}}}\right)^{2}=\frac{I_{\mathcal{B}_{i}}}{I_{\mathcal{B}_{i-1}}} \frac{I_{\mathcal{B}_{i}}}{I_{\mathcal{B}_{i-1}}}=\frac{I_{\mathcal{B}_{i}}^{2}+I_{\mathcal{B}_{i-1}}}{I_{\mathcal{B}_{i-1}}}=\frac{I_{\mathcal{B}_{i}}}{I_{\mathcal{B}_{i-1}}}
$$

Therefore, we have a new definition

Definition 2.5. Let $\left\{\mathcal{B}_{i}\right\}_{i \geq 0}$ be an exhaustive filtration of $\mathcal{C}$ into subcategories. Thus, $\mathcal{C}$ is said to be quasi-hereditary with respect to $\left\{\mathcal{B}_{i \geq 0}\right\}$ if $I_{\mathcal{B}_{i}} / I_{\mathcal{B}_{i-1}}$ is heredity in the category $\mathcal{C} / I_{\mathcal{B}_{i-1}}$.

Before announcing the main theorem of this section we need the following.

Lemma 2.6. Consider a pair of subcategories $\mathcal{B} \subset \mathcal{B}^{\prime} \subset \mathcal{C}$ which are closed under direct summands. Then $I_{\mathcal{B}^{\prime}}(-, X) / I_{\mathcal{B}}(-, X)$ is a projective $\mathcal{C} / I_{\mathcal{B}}$-module if and only if it is isomorphic to $\frac{\mathcal{C}(-, E)}{I_{\mathcal{B}}(-, E)}, E \in \mathcal{B}^{\prime}$.

Proof. First, assume that there exists an isomorphism of $\mathcal{C} / I_{\mathcal{B}}$-modules

$$
\varphi: \frac{\mathcal{C}(-, E)}{I_{\mathcal{B}}(-, E)} \rightarrow \frac{I_{\mathcal{B}^{\prime}}(-, X)}{I_{\mathcal{B}}(-, X)} .
$$

Proving that $E \in \mathcal{B}^{\prime}$ is then sufficient.

Let $E^{\prime}$ be an indecomposable summand of $E$ and $E^{\prime} \stackrel{j}{\rightarrow} E \stackrel{p}{\rightarrow} E^{\prime}$ be the canonical inclusion and projection respectively.

Set $\varphi_{E^{\prime}}\left(j+I_{\mathcal{B}}\left(E^{\prime}, E\right)\right)=\left(E^{\prime} \stackrel{f}{\rightarrow} B \stackrel{g}{\rightarrow} X\right)+I_{\mathcal{B}}\left(E^{\prime}, X\right) \in \frac{I_{\mathcal{B}^{\prime}}\left(E^{\prime}, X\right)}{I_{\mathcal{B}}\left(E^{\prime}, X\right)}$, with $B \in \mathcal{B}^{\prime}$. Thus, we have the following commutative diagram:

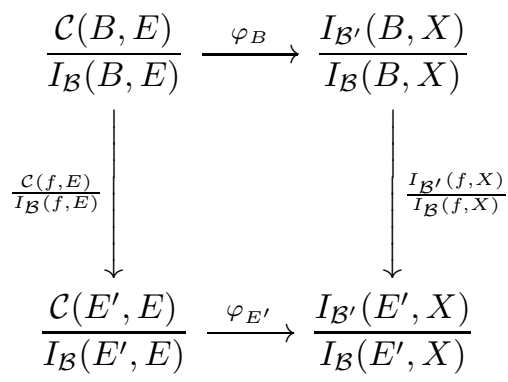

We have $\frac{I_{\mathcal{B}^{\prime}}(f, E)}{I_{\mathcal{B}}(f, E)}\left(g+I_{\mathcal{B}}(B, X)\right)=\varphi_{E^{\prime}}\left(j+I_{\mathcal{B}}\left(E^{\prime}, E\right)\right)$. Let $h \in \mathcal{C}(B, E)$ such that $\varphi_{B}\left(h+I_{\mathcal{B}}(B, E)\right)=g+I_{\mathcal{B}}(B, X)$. Since the diagram is commutative, it follows that $\frac{I_{\mathcal{B}^{\prime}}(f, E)}{I_{\mathcal{B}}\left(f, E_{j}\right)}\left(h+I_{\mathcal{B}}(B, E)\right)=h f+I_{\mathcal{B}}\left(E^{\prime}, E\right)=j+I_{\mathcal{B}}\left(E^{\prime}, E\right)$. Thus, $1_{E^{\prime}}=p j=p h f$ and, $E^{\prime}$ is a direct summand of $B$, i.e, $E^{\prime} \in \mathcal{B}^{\prime}$.

Theorem 2.7. Let $\left\{\mathcal{B}_{i}\right\}_{i \geq 0}$ be an exahustive filtration of $\mathcal{C}$ into subcategories which are closed under direct summands. Then, $\mathcal{C}$ is quasi-hereditary with respect to $\left\{\mathcal{B}_{i}\right\}_{i \geq 0}$ if and only if the following holds.

(i) $\operatorname{rad} \mathcal{C}\left(E, E^{\prime}\right)=I_{\mathcal{B}_{j-1}}\left(E, E^{\prime}\right)$ for all pairs of indecomposable $E, E^{\prime} \in \mathcal{B}_{j}$ such that none of them are a direct summand of any object in $\mathcal{B}_{j-1}$. 
(ii) For all $X \in \mathcal{C}$ and $j \geq 1$, there exists an exact sequence

$$
\mathcal{C}\left(-, E_{j-1}\right) \rightarrow \mathcal{C}\left(-, E_{j}\right) \rightarrow I_{\mathcal{B}_{j}}(-, X) \rightarrow 0,
$$

with $E_{j} \in \mathcal{B}_{j}$ and $E_{j-1} \in \mathcal{B}_{j-1}$.

Proof. Given the filtration $\left\{\mathcal{B}_{i}\right\}_{i \geq 0}$, we prove that $I_{\mathcal{B}_{i}} / I_{\mathcal{B}_{i-1}}$ is heredity in $\mathcal{C} / I_{\mathcal{B}_{i-1}}$ if and only if (i) and (ii) hold.

(i) a) $\frac{I_{\mathcal{B}_{j}}}{I_{\mathcal{B}_{j-1}}} \operatorname{rad}\left(\frac{\mathcal{C}(-, ?)}{I_{\mathcal{B}_{j-1}}}\right) \frac{I_{\mathcal{B}_{j}}}{I_{\mathcal{B}_{j-1}}}=0$ if and only if $\frac{I_{\mathcal{B}_{j}}}{I_{\mathcal{B}_{j-1}}}\left[\frac{\operatorname{radC}(-, ?)+I_{\mathcal{B}_{j-1}}}{I_{\mathcal{B}_{j-1}}}\right] \frac{I_{\mathcal{B}_{j}}}{I_{\mathcal{B}_{j-1}}}=0$ if and only if $\frac{I_{\mathcal{B}_{j}}\left(\operatorname{radC}(-, \text { ? })+I_{\mathcal{B}_{j-1}}\right) I_{\mathcal{B}_{j}}+I_{\mathcal{B}_{j-1}}}{I_{\mathcal{B}_{j-1}}}=0$ if and only if

$$
I_{\mathcal{B}_{j}} \operatorname{radC}(-, ?) I_{\mathcal{B}_{j}}+I_{\mathcal{B}_{j-1}} I_{\mathcal{B}_{j}}+I_{\mathcal{B}_{j-1}}=I_{\mathcal{B}_{j-1}} .
$$

On the other hand, we have $I_{\mathcal{B}_{j-1}}=I_{\mathcal{B}_{j-1}}^{2} \subset I_{\mathcal{B}_{j-1}} I_{\mathcal{B}_{j}} \subset I_{\mathcal{B}_{j-1}}$, i.e, $I_{\mathcal{B}_{j-1}}=I_{\mathcal{B}_{j-1}} I_{\mathcal{B}_{j}}$. Thus, equation (2) implies

$$
I_{\mathcal{B}_{j}} \operatorname{rad} \mathcal{C}(-, ?) I_{\mathcal{B}_{j}} \subseteq I_{\mathcal{B}_{j-1}}
$$

b) Now we show that $I_{\mathcal{B}_{j}} \operatorname{rad} \mathcal{C}(-, ?) I_{\mathcal{B}_{j}} \subseteq I_{\mathcal{B}_{j-1}}$ if and only if $\operatorname{rad} \mathcal{C}\left(E, E^{\prime}\right)=$ $I_{\mathcal{B}_{j-1}}\left(E, E^{\prime}\right)$ for all pairs of indecomposable $E, E^{\prime} \in \mathcal{B}_{j}$ such that none of them are a direct summand of any object in $\mathcal{B}_{j-1}$. Assume $I_{\mathcal{B}_{j}} \operatorname{rad} \mathcal{C}\left(-\right.$, ?) $I_{\mathcal{B}_{j}} \subseteq I_{\mathcal{B}_{j-1}}$. Let $t \in$ $\operatorname{rad} \mathcal{C}\left(E, E^{\prime}\right)$, then $t=1_{E^{\prime}} t 1_{E} \in I_{\mathcal{B}_{j}}(E, E) \operatorname{rad} \mathcal{C}\left(E, E^{\prime}\right) I_{\mathcal{B}_{j}}\left(E^{\prime}, E^{\prime}\right) \subseteq I_{\mathcal{B}_{j-1}}\left(E, E^{\prime}\right)$. On the other hand, if $f \in I_{\mathcal{B}_{j-1}}\left(E, E^{\prime}\right)$ then there exists $B \in \mathcal{B}_{j-1}$ for which $f=E \stackrel{u}{\rightarrow} B \stackrel{v}{\rightarrow} E^{\prime}$. Thus $f$ is not an isomorphism. Otherwise, there exists $g: E^{\prime} \rightarrow E$ such that $f g=1_{E^{\prime}}$. Therefore $v u g=1_{E^{\prime}}$, i.e, $E^{\prime}$ is a direct summand of $B$; hence $E^{\prime} \in \mathcal{B}_{j-1}$. This contradiction implies that $I_{\mathcal{B}_{j-1}}\left(E, E^{\prime}\right) \subset \operatorname{rad} \mathcal{C}\left(E, E^{\prime}\right)$.

Conversely, assume $\operatorname{rad} \mathcal{C}\left(E, E^{\prime}\right)=I_{\mathcal{B}_{j-1}}\left(E, E^{\prime}\right)$. Let $f \in I_{\mathcal{B}}(X, Y) \operatorname{rad} \mathcal{C}(Y, Z) I_{\mathcal{B}}(Z, W)$. If $f=r s t$ with $r \in I_{\mathcal{B}_{j}}(X, Y), t \in \operatorname{radC}(Y, Z)$ and $s \in I_{\mathcal{B}_{j}}(Z, W)$, then we can write $r: X \rightarrow \coprod B_{i} \rightarrow Y$ and $s: Z \rightarrow \coprod B_{j} \rightarrow W$, where the terms in the middle are finite sums of indecomposable objects in $\mathcal{B}$. It follows that the induced maps $B_{i} \rightarrow Y \stackrel{t}{\rightarrow} Z \rightarrow B_{j}$ are all in $\operatorname{rad} \mathcal{C}\left(B_{i}, B_{j}\right)=I_{\mathcal{B}_{j-1}}\left(B_{i}, B_{j}\right)$, and therefore $f \in I_{\mathcal{B}_{j-1}}(X, W)$.

(ii) First we prove the sufficiency by induction. The case $j=1$ has been proved in Lemma 2.1 because $I_{\mathcal{B}_{0}}=0$.

Assume that the statement is true for $j-1$ and that $\frac{I_{\mathcal{B}_{j}}(-, X)}{I_{\mathcal{B}_{j-1}}(-, X)}$ is a finitely projective $\mathcal{C} / I_{\mathcal{B}_{j-1}}$ - module. We have then an isomorphism $\frac{\mathcal{C}\left(-, E_{j}\right)}{I_{\mathcal{B}_{j-1}}\left(-, E_{j}\right)} \cong \frac{I_{\mathcal{B}_{j}}(-, X)}{I_{\mathcal{B}_{j-1}}(-, X)}$ with $E_{j} \in \mathcal{B}_{j}$ by Lemma 2.6. Therefore, we have a pushout diagram:

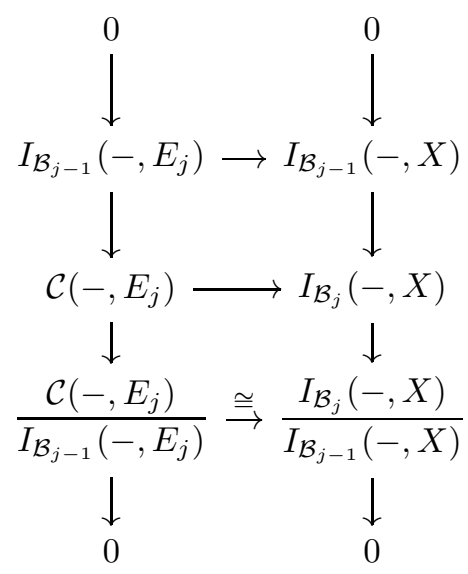

which induces the following exact sequence

$$
I_{\mathcal{B}_{j-1}}\left(-, E_{j}\right) \rightarrow \mathcal{C}\left(-, E_{j}\right) \coprod I_{\mathcal{B}_{j-1}}(-, X) \stackrel{\pi}{\rightarrow} I_{\mathcal{B}_{j}}(-, X) \rightarrow 0
$$


THE AUSLANDER-REITEN COMPONENTS SEEN AS QUASI-HEREDITARY CATEGORIES 9

On the other hand, by induction hypothesis, there are exact sequences

$$
\begin{array}{r}
\mathcal{C}\left(-, E_{j-2}^{\prime}\right) \rightarrow \mathcal{C}\left(-, E_{j-1}^{\prime}\right) \stackrel{p}{\rightarrow} I_{\mathcal{B}_{j-1}}(-, X) \rightarrow 0, \\
\mathcal{C}\left(-, E_{j-2}^{\prime \prime}\right) \rightarrow \mathcal{C}\left(-, E_{j-1}^{\prime \prime}\right) \stackrel{q}{\rightarrow} I_{\mathcal{B}_{j-1}}\left(-, E_{j}\right) \rightarrow 0,
\end{array}
$$

with $E_{j-1}, E_{j-1}^{\prime} \in \mathcal{B}_{j-1}$ and $E_{j-2}, E_{j-2}^{\prime \prime} \in \mathcal{B}_{j-2}$.

Thus, we have the following commutative diagram:

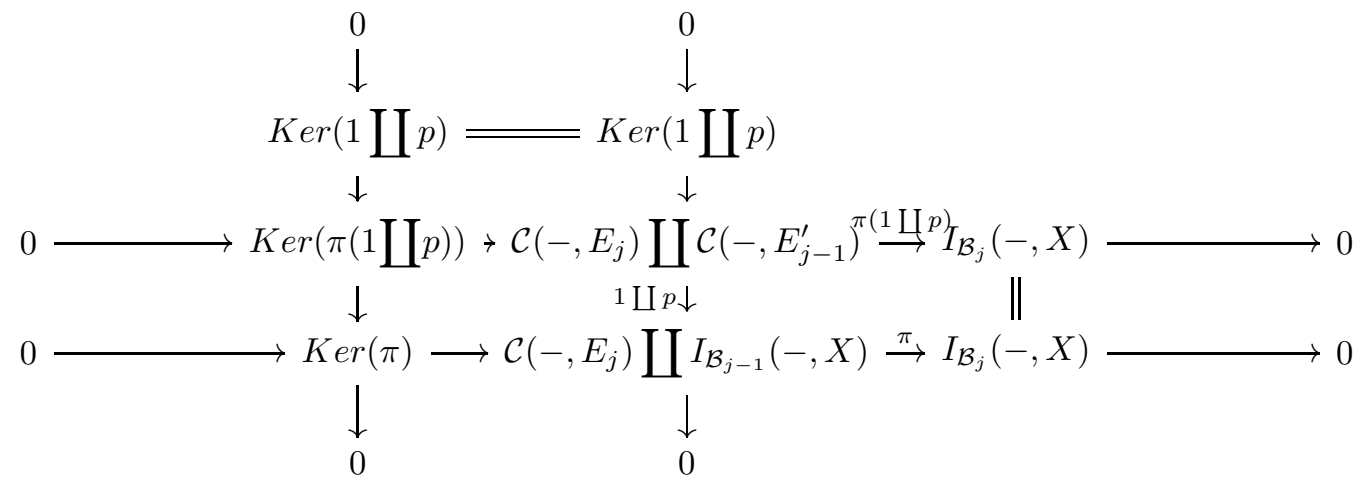

From the epimorphisms $I_{\mathcal{B}_{j-1}}\left(-, E_{j}\right) \rightarrow \operatorname{Ker}(\pi) \rightarrow 0$ and $\mathcal{C}\left(-, E_{j-2}^{\prime}\right) \rightarrow \operatorname{Ker}(p)=$ $\operatorname{Ker}(1 \coprod p) \rightarrow 0$, the exact sequences (4) and the horseshoe lemma, we have an epimorphism $\mathcal{C}\left(-, E_{j-2}^{\prime}\right) \coprod \mathcal{C}\left(-, E_{j-1}^{\prime \prime}\right) \rightarrow \operatorname{Ker}(\pi(1 \coprod p)) \rightarrow 0$. In this way, we have an exact sequence:

$$
\mathcal{C}\left(-, E_{j-2}^{\prime}\right) \coprod \mathcal{C}\left(-, E_{j-1}^{\prime \prime}\right) \rightarrow \mathcal{C}\left(-, E_{j}\right) \coprod \mathcal{C}\left(-, E_{j-1}^{\prime}\right) \stackrel{\pi(1 \amalg p)}{\longrightarrow} I_{\mathcal{B}_{j}}(-, X) \rightarrow 0
$$

It only remains to prove necessity. First, observe that $\frac{\mathcal{C}\left(?, E_{j-1}\right)}{I_{\mathcal{B}_{j-1}}\left(?, E_{j-1}\right)} \cong 0$ because $E_{j-1}$ lies in $\mathcal{B}_{j-1}$ and therefore $\mathcal{C}\left(-, E_{j-1}\right)=I_{\mathcal{B}_{j-1}}\left(-, E_{j-1}\right)$. After applying $-\otimes$

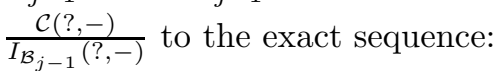

$$
\mathcal{C}\left(-, E_{j-1}\right) \rightarrow \mathcal{C}\left(-, E_{j}\right) \rightarrow I_{\mathcal{B}_{j}}(-, X) \rightarrow 0
$$

and using the isomorphisms $\mathcal{C}\left(-, E_{j-1}\right) \otimes \frac{\mathcal{C}(?,-)}{I_{\mathcal{B}_{j-1}}(?,-)} \cong \frac{\mathcal{C}\left(?, E_{j-1}\right)}{I_{\mathcal{B}_{j-1}}\left(?, E_{j-1}\right)} \cong 0$ and $\mathcal{C}\left(-, E_{j}\right) \otimes \frac{\mathcal{C}(?,-)}{I_{\mathcal{B}_{j-1}}(?,-)} \cong \frac{\mathcal{C}\left(?, E_{j}\right)}{I_{\mathcal{B}_{j-1}}\left(?, E_{j}\right)}$, we obtain the isomorphism:

$$
\frac{\mathcal{C}\left(?, E_{j}\right)}{I_{\mathcal{B}_{j-1}}\left(?, E_{j}\right)} \cong I_{\mathcal{B}_{j}}(-, X) \otimes \frac{\mathcal{C}(?,-)}{I_{\mathcal{B}_{j-1}}(?,-)}
$$

Finally, we prove that $I_{\mathcal{B}_{j}}(-, X) \otimes \frac{\mathcal{C}(?,-)}{I_{\mathcal{B}_{j-1}}(?,-)} \cong \frac{I_{\mathcal{B}_{j}}(?, X)}{I_{\mathcal{B}_{j-1}}(?, X)}$. First, we prove the following isomorphism:

$$
I_{\mathcal{B}_{j-1}}(?, X) \cong I_{\mathcal{B}_{j}}(-, X) \otimes I_{\mathcal{B}_{j-1}}(?,-) .
$$

Indeed after appliying $I_{\mathcal{B}_{j}}(-, X) \otimes-$ to the exact sequence of $\mathcal{C}^{o p}$-modules

$$
\mathcal{C}\left(E_{j-2}^{\prime},-\right) \rightarrow \mathcal{C}\left(E_{j-1}^{\prime},-\right) \rightarrow I_{\mathcal{B}_{j-1}}(?,-) \rightarrow 0
$$

and using the isomorphisms $I_{\mathcal{B}_{j}}(-, X) \otimes \mathcal{C}\left(E_{j-2}^{\prime},-\right) \cong I_{\mathcal{B}_{j}}\left(E_{j-2}^{\prime}, X\right)=\mathcal{C}\left(E_{j-2}^{\prime}, X\right)$ and $I_{\mathcal{B}_{j}}(-, X) \otimes \mathcal{C}\left(E_{j-1}^{\prime},-\right) \cong I_{\mathcal{B}_{j}}\left(E_{j-1}^{\prime}, X\right)=\mathcal{C}\left(E_{j-1}^{\prime}, X\right)$ ( because $E_{j-1}^{\prime}, E_{j-2}^{\prime} \in$ $\left.\mathcal{B}_{j}\right)$, we conclude that $I_{\mathcal{B}_{j}}(-, X) \otimes I_{\mathcal{B}_{j-1}}(?,-) \cong I_{\mathcal{B}_{j-1}}(?, X)$. 
On the other hand, after appliying $I_{\mathcal{B}_{j}}(-, X) \otimes-$ to the exact sequence $0 \rightarrow$ $I_{\mathcal{B}_{j-1}}(?,-) \rightarrow \mathcal{C}(?,-) \rightarrow \frac{\mathcal{C}(?,-)}{I_{\mathcal{B}_{j-1}(?,-)}} \rightarrow 0$ and by using the isomorphism (5) and $I_{\mathcal{B}_{j}}(-, X) \otimes \mathcal{C}(?,-) \cong I_{\mathcal{B}_{j}}(?, X)$, we obtain the following commutative diagram:

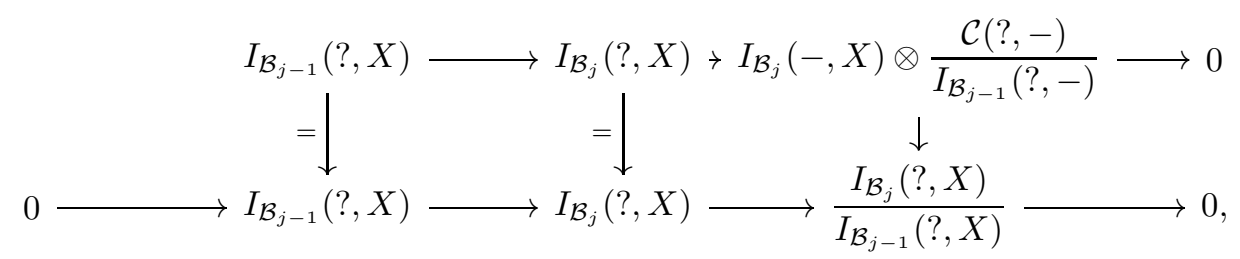

and the isomorphism follows.

2.3. The standard and costandard subcategories of $\mathcal{C}$-modules. Let $\left\{\mathcal{B}_{j}\right\}_{j \geq 0}$ be a exhaustive filtration of $\mathcal{C}$ into subcategories which are closed under direct summands:

$$
0=\mathcal{B}_{0} \subset \mathcal{B}_{1} \subset \cdots \subset \mathcal{B}_{j-1} \subset \mathcal{B}_{j} \subset \cdots \subset \mathcal{C}
$$

In the rest of this section, all the filtrations $\left\{\mathcal{B}_{j}\right\}_{j \geq 0}$ we consider are filtrations into subcategories that are closed under direct summands.

We introduce the concept of standard and costandard subcategories of $\mathcal{C}$-modules with respect to the given filtration $\left\{\mathcal{B}_{j \geq 0}\right\}_{j \geq 0}$.

Definition 2.8. Dlab] The sequence

$$
\Delta=\Delta_{\mathcal{C}}=\{\Delta(j): j \geq 1\}
$$

of (contravariant) standard subcategories with respect to a given filtration $\left\{\mathcal{B}_{j}\right\}_{j \geq 0}$ is given by

$$
\Delta(j)=\left\{\frac{\mathcal{C}(-, E)}{I_{\mathcal{B}_{j-1}}(-, E)} \mid E \in \mathcal{B}_{j}\right\}
$$

Similarly, there is a sequence

$$
\Delta^{\circ}=\Delta_{\mathcal{C}}^{\circ}=\left\{\Delta^{\circ}(j): j \geq 1\right\}
$$

of covariant standard subcategories $\Delta^{\circ}(j)=\left\{\frac{\mathcal{C}(E,-)}{I_{\mathcal{B}_{j-1}}(E,-)} \mid E \in \mathcal{B}_{j}\right\}$ and the sequence of its duals, the (contravariant) costandard subcategories $\nabla=\nabla_{\mathcal{C}}=\left\{D \Delta^{\circ}(j): j \geq\right.$ 1) where

$$
D \Delta^{\circ}(j)=\left\{D \frac{\mathcal{C}(E,-)}{I_{\mathcal{B}_{j-1}}(E,-)} \mid E \in \mathcal{B}_{j}\right\} .
$$

Call the sequence $\Delta$ Schurian if every $\Delta_{X}(j)$ is Schurian, in other words $\operatorname{End}\left(\Delta_{X}(j)\right)$ is a division algebra for all $j \geq 1$, with $X \in \mathcal{B}_{j}$ indecomposable.

Let us describe some of the basic propierties of the standard and costandard subcategories. In the rest of this subsection we asumme that $\mathcal{C}$ is a quasi-hereditary category with respect to the filtration (6) .

Lemma 2.9. 1) If $\operatorname{Hom}(\Delta(i), \Delta(j)) \neq 0$ implies $i \geq j$. 2) $\operatorname{Ext}^{1}(\Delta(i), \Delta(j)) \neq 0$ implies $i>j$. 3) The sequence $\Delta$ is Schurian

Proof. Set $\Delta_{Y}(j)=\mathcal{C}(-, Y) / I_{\mathcal{B}_{j-1}}(-, Y), Y \in \mathcal{B}_{j}$ and $\Delta_{X}(i)=\mathcal{C}(-, X) / I_{\mathcal{B}_{i-1}}(-, X)$, $X \in \mathcal{B}_{i}$. After applying $\left(-, \Delta_{E}(j)\right)$ to the sequence $0 \rightarrow I_{\mathcal{B}_{i-1}}(-, X) \rightarrow \mathcal{C}(-, X) \rightarrow$ $\Delta_{X}(i) \rightarrow 0$, we get by the long homology sequence the exact sequence

$$
\begin{array}{r}
0 \rightarrow\left(\Delta_{X}(i), \Delta_{Y}(j)\right) \rightarrow\left(\mathcal{C}(-, X), \Delta_{Y}(j)\right) \rightarrow\left(I_{\mathcal{B}_{i-1}}(-, X), \Delta_{Y}(j)\right) \\
\rightarrow \operatorname{Ext}^{1}\left(\Delta_{X}(i), \Delta_{Y}(j)\right) \rightarrow \operatorname{Ext}^{1}\left(\left(\mathcal{C}(-, X), \Delta_{Y}(j)\right)=0 .\right.
\end{array}
$$


1) Let us assume $i<j$, then $i \leq j-1$ and $\mathcal{B}_{i} \subset \mathcal{B}_{j-1}$. It is enough to prove that $\left(\mathcal{C}(-, X), \Delta_{Y}(j)\right)=0$. Since $X$ is an object in $\mathcal{B}_{i}$, it follows that $X$ is an object in $\mathcal{B}_{j-1}$ and

$$
\left(\mathcal{C}(-, X), \Delta_{Y}(j)\right)=\frac{\mathcal{C}(X, Y)}{I_{\mathcal{B}_{j-1}}(X, Y)}=0 .
$$

Therefore, (7) implies $\operatorname{Hom}\left(\Delta_{X}(i), \Delta_{Y}(j)\right)=0$.

2) Let us assume $i \leq j$, then $\mathcal{B}_{i-1} \subset \mathcal{B}_{j-1}$. It is enough to prove that $\left(I_{\mathcal{B}_{i}}(-, X), \Delta_{Y}(j)\right)=$ 0 . There is an exact sequence

$$
\left(-, E_{i-2}\right) \rightarrow\left(-, E_{i-1}\right) \rightarrow I_{\mathcal{B}_{i-1}}(-, X) \rightarrow 0,
$$

with $E_{i-1} \in \mathcal{B}_{i-1}, E_{i-2} \in \mathcal{B}_{i-2}$.

After applying $\left(-, \Delta_{Y}(j)\right)$ to the sequence (8), we get the exact sequence

$$
0 \rightarrow\left(I_{\mathcal{B}_{i}}(-, X), \Delta_{Y}(j)\right) \rightarrow\left(\left(-, E_{i-1}\right), \Delta_{Y}(j)\right) \rightarrow\left(\left(-, E_{i-2}\right), \Delta_{Y}(j)\right)
$$

Since $E_{i-1} \in \mathcal{B}_{i-1} \subset \mathcal{B}_{j-1}$, we have $\left(\mathcal{C}\left(-, E_{i-1}\right), \Delta_{Y}(j)\right)=0$, which implies $\left(I_{\mathcal{B}_{i}}(-, X), \Delta_{Y}(j)\right)=0$. Thus (7) implies $\operatorname{Ext}^{1}\left(\Delta_{X}(i), \Delta_{Y}(j)\right)=0$.

3) Let $X \in \mathcal{B}_{i}-\mathcal{B}_{i-1}$ indescomposable. It follows from the exact sequence (7) and part 2) of Lemma 2.9 that $\left(\Delta_{X}(i), \Delta_{X}(i)\right) \cong\left(\mathcal{C}(-, X), \Delta_{X}(i)\right)=\frac{\mathcal{C}(X, X)}{I_{\mathcal{B}_{i-1}}(X, X)}$, however, by Theorem 2.7 we have $I_{\mathcal{B}_{i-1}}(X, X) \cong \operatorname{rad}(X, X)$. Thus $\operatorname{End}\left(\Delta_{X}(i)\right)=$ $\operatorname{End}_{\mathcal{C}}(X) / \operatorname{rad}\left(\operatorname{End}_{\mathcal{C}}(X)\right)$ is division algebra.

Lemma 2.10. 1) If $\operatorname{Hom}(\Delta(i), \nabla(j)) \neq 0$ implies $i=j$. 2) $\operatorname{Ext}^{1}(\Delta(i), \nabla(j))=0$ for all $i, j$. 2') $\operatorname{Tor}_{1}\left(\Delta(i), \Delta^{\circ}(j)\right)=0$ for all $i, j$.

Proof. Set $\nabla_{Y}(j)=D\left(\mathcal{C}(Y,-) / I_{\mathcal{B}_{j-1}}(Y,-)\right), Y \in \mathcal{B}_{j}$ and $\Delta_{X}(i)=\mathcal{C}(-, X) / I_{\mathcal{B}_{i-1}}(-, X)$, $X \in \mathcal{B}_{i}$.

After applying $\left(-, \nabla_{Y}(j)\right)$ to the sequence $0 \rightarrow I_{\mathcal{B}_{i-1}}(-, X) \rightarrow \mathcal{C}(-, X) \rightarrow$ $\Delta_{X}(i) \rightarrow 0$, we get by the long homology sequence, the exact sequence

(9) $\quad 0 \rightarrow\left(\Delta_{X}(i), \nabla_{Y}(j)\right) \rightarrow\left(\mathcal{C}(-, X), \nabla_{Y}(j)\right) \rightarrow\left(I_{\mathcal{B}_{i-1}}(-, X), \nabla_{Y}(j)\right)$

$$
\rightarrow \operatorname{Ext}^{1}\left(\Delta_{X}(i), \nabla_{Y}(j)\right) \rightarrow \operatorname{Ext}^{1}\left(\left(\mathcal{C}(-, X), \nabla_{Y}(j)\right)=0 .\right.
$$

After applying $\left(\Delta_{X}(i),-\right)$ to the sequence $0 \rightarrow \nabla_{Y}(j) \rightarrow D \mathcal{C}(Y,-) \rightarrow D I_{\mathcal{B}_{j-1}}(Y,-) \rightarrow$ 0 , we get by the long homology sequence the exact sequence

(10) $0 \rightarrow\left(\Delta_{X}(i), \nabla_{Y}(j)\right) \rightarrow\left(\Delta_{X}(i), D \mathcal{C}(Y,-)\right) \rightarrow\left(\Delta_{X}(i), D I_{\mathcal{B}_{i-1}}(Y,-)\right)$

$$
\rightarrow \operatorname{Ext}^{1}\left(\Delta_{X}(i), \nabla_{Y}(j)\right) \rightarrow \operatorname{Ext}^{1}\left(\Delta_{X}(i), D \mathcal{C}(Y,-)\right)=0 .
$$

1) First, let us assume $i<j$, then $i \leq j-1$ and $\mathcal{B}_{i} \subset \mathcal{B}_{j-1}$. Since $X \in \mathcal{B}_{i} \subset \mathcal{B}_{j-1}$, we have

$$
(\mathcal{C}(-, X), \nabla(j)) \cong D\left(\frac{\mathcal{C}(Y, X)}{I_{\mathcal{B}_{j-1}}(Y, X)}\right)=0
$$

It follows from (9) and (11) that $\operatorname{Hom}\left(\Delta_{X}(i), \nabla_{Y}(j)\right)=0$ when $i<j$.

On the other hand, let us assume $j<i$, then $j \leq i-1$ and $\mathcal{B}_{j} \subset \mathcal{B}_{i-1}$. Since $Y \in \mathcal{B}_{j} \subset \mathcal{B}_{i-1}$, we have

$$
(\Delta(i), D \mathcal{C}(Y,-)) \cong(\mathcal{C}(Y,-), D \Delta(i)) \cong D \frac{\mathcal{C}(Y, X)}{I_{\mathcal{B}_{i-1}}(Y, X)} \cong 0
$$

It follows from (10) and (35) that $\operatorname{Hom}\left(\Delta_{X}(i), \nabla_{Y}(j)\right)=0$ when $j<i$

2) Assume that $i \leq j$, then $\mathcal{B}_{i} \subset \mathcal{B}_{j}$. There is an exact sequence

$$
\left(-, E_{i-2}\right) \rightarrow\left(-, E_{i-1}\right) \rightarrow I_{\mathcal{B}_{i-1}}(-, X) \rightarrow 0
$$


with $E_{i-1} \in \mathcal{B}_{i-1}, E_{i-2} \in \mathcal{B}_{i-2}$. After applying $(-, \Delta(j))$ to the above sequence we get a monomorphism

$$
0 \rightarrow\left(I_{\mathcal{B}_{i-1}}(-, X), \nabla_{Y}(j)\right) \rightarrow\left(\mathcal{C}\left(-, E_{i-1}\right), \nabla_{Y}(j)\right) \cong D \frac{\mathcal{C}\left(Y, E_{i-1}\right)}{I_{\mathcal{B}_{j-1}}\left(Y, E_{i-1}\right)}=0
$$

It follows from (9) that $\operatorname{Ext}^{1}\left(\Delta_{X}(i), \nabla_{Y}(j)\right)=0$ when $i \leq j$.

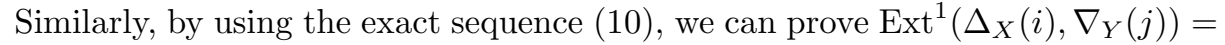
0 when $j \leq i$.

2.4. Trace filtrations. The categories $\mathcal{F}(\Delta)$ and $\mathcal{F}(\nabla)$. Throughout this subsection $\mathcal{C}$ will be a Hom-finite Krull-Schmidt quasi-hereditary category $K$-category $\mathcal{C}$ with an exhaustive filtration $\left\{\mathcal{B}_{i}\right\}_{i \geq 0}$

$$
0=\mathcal{B}_{0} \subset \mathcal{B}_{1} \subset \cdots \subset \mathcal{B}_{j-1} \subset \mathcal{B}_{j} \subset \cdots \subset \mathcal{C} .
$$

We remember that under these conditions finitely presented functors have projective covers [MVO2]. In this part we introduce some special subcategories related to the described filtrations in Theorem 2.7. First we assume that the filtration $\left\{\mathcal{B}_{i}\right\}_{i \geq 0}$ is not necessarily finite and we obtain general results. After we add the condition that the filtration is finite, obtaining in this way results similar to those appearing in [Rin2].

For every $\mathcal{C}$-module $F$, there is an associated filtration.

Definition 2.11. Given a $\mathcal{C}$-module $F$, define its trace filtration (with respect to $\left.\left\{\mathcal{B}_{j}\right\}_{j \geq 0}\right)$ by

$$
0=F^{(0)} \subset F^{(1)} \subset \cdots F^{(j-1)} \subset F^{(j)} \subset \cdots,
$$

where $F^{(j)}=\operatorname{Tr}_{\{\mathcal{C}(-, E)\}_{E \in \mathcal{B}_{j}}} F$ and $F=\bigcup_{j \geq 0} F^{(j)}$.

In this part, we shall focus on the $\mathcal{C}$-modules $F$ whose trace filtrations satisfy the condition that $F^{(i)} / F^{(i-1)}$ is a finite direct sum of objects from the category $\Delta(i)$ for every $i \geq 1$. In this case we say that these $\mathcal{C}$-modules possess a $\Delta$-filtration. Denote the full subcategory of all $\mathcal{C}$-modules with $\Delta$-filtration by $\mathcal{F}(\Delta)$.

First we give some descriptions of the categories of $\mathcal{C}$-modules with a $\Delta$-filtration in the case where the filtration $\left\{\mathcal{B}_{i}\right\}_{i \geq 0}$. Finally, we consider the finite case, obtaining a characterization as that given in Dlab. In order to do this, we start with a list of lemmas.

Lemma 2.12. Let $F \in \mathcal{F}(\Delta)$. Then

(i) For all $i \geq 0, F^{(i)}$ has a presentation

$$
\mathcal{C}\left(-, E_{i-1}\right) \rightarrow \mathcal{C}\left(-, E_{i}\right) \rightarrow F^{(i)} \rightarrow 0
$$

with $E_{i-1} \in \mathcal{B}_{i-1}$ and $E_{i} \in \mathcal{B}_{i}$.

(ii) $F^{(i)} \cong \mathcal{C} \otimes_{\mathcal{B}_{i}}\left(\left.F\right|_{\mathcal{B}_{i}}\right)$, (see Proposition A.3.2 in Dlab]).

Proof. We prove (i) by induction. Since $I_{\mathcal{B}_{0}}(-, E)=0$ for all $E \in \mathcal{B}_{1}$, we have $\Delta(1)=\left\{\mathcal{C}(-, E) \mid E \in \mathcal{B}_{1}\right\}$. Let $F \in \mathcal{F}(\Delta)$, then $F^{(0)}=0$, and $F^{(1)} / F^{(0)}=F^{(1)}$ is a finite direct sum of objects of $\Delta(1)$. Assume that there exists an exact sequence for $F^{(i-1)}$ :

$$
\mathcal{C}\left(-, E_{i-2}\right) \rightarrow \mathcal{C}\left(-, E_{i-1}\right) \rightarrow F^{(i-1)} \rightarrow 0 .
$$


On the other hand we have an exact sequence

$$
0 \rightarrow F^{(i-1)} \rightarrow F^{(i)} \rightarrow \frac{F^{(i)}}{F^{(i-1)}} \rightarrow 0 .
$$

Since $F \in \mathcal{F}(\Delta)$, it follows that $\frac{F^{(i)}}{F^{(i-1)}}$ is a finite direct sum of objects $\Delta_{E}(i)$, $E \in \mathcal{B}_{i}$. We write $\Delta_{E}(i)=\frac{\mathcal{C}(-, E)}{I_{\mathcal{B}_{i-1}}(-, E)}$. Thus, we have an exact sequence for all $E \in \mathcal{B}_{i}$

$$
0 \rightarrow I_{\mathcal{B}_{i-1}}(-, E) \rightarrow \mathcal{C}(-, E) \rightarrow \frac{\mathcal{C}(-, E)}{I_{\mathcal{B}_{i-1}}(-, E)} \rightarrow 0
$$

Furthermore, there is an exact sequence

$$
\mathcal{C}\left(-, E_{i-1}^{\prime}\right) \rightarrow \mathcal{C}\left(-, E_{i-1}^{\prime}\right) \rightarrow I_{\mathcal{B}_{i-1}}(-, E) \rightarrow 0,
$$

by Theorem 2.7. Thus, we have an exact sequence

$$
\mathcal{C}\left(-, E_{i-1}^{\prime}\right) \rightarrow \mathcal{C}(-, E) \rightarrow \Delta_{E}(i) \rightarrow 0
$$

for all $E \in \mathcal{B}_{i}$. The desired resolution is obtained from the exact sequences (14), (15) and (16).

(ii) First observe that $\left(\frac{F}{F^{(i)}}\right)^{(i)}=0$. Thus $\left((-, E), \frac{F}{F^{(i)}}\right)=0$ for all $E \in \mathcal{B}_{i}$, and therefore $\frac{F}{F^{(i)}}(E)=\left.\frac{F}{F^{(i)}}\right|_{\mathcal{B}_{i}}(E)=\left((-, E), \frac{F}{F^{(i)}}\right)=0$, for all $E \in \mathcal{B}_{i}$, i.e, $\left.\frac{F}{F^{(i)}}\right|_{\mathcal{B}_{i}}=0$. After then appliying the exact functor $\left.\right|_{\mathcal{B}_{i}}$ to the exact sequece $0 \rightarrow F^{(i)} \rightarrow F \rightarrow$ $\frac{F}{F^{(i)}} \rightarrow 0$, we obtain

$$
\left.\left.F^{(i)}\right|_{\mathcal{B}_{i}} \cong F\right|_{\mathcal{B}_{i}}
$$

On the other hand, it follows from (13) that $F^{(i)}$ is projectively presented over $\mathcal{B}_{i}$. Thus, $\mathcal{C} \otimes_{\mathcal{B}_{i}}\left(\left.F^{(i)}\right|_{\mathcal{B}_{i}}\right) \cong F^{(i)}$ (see [Au]) and by using (17) we obtain (ii).

Lemma 2.13. Assume that $F \in \mathcal{F}(\Delta)$. Then the following holds:

(i) $F$ is locally finite.

(ii) $F$ is finitely presented if and only if $F=F^{(i)}$ for some $i \in \mathbb{N}$.

(iii) If the filtartion $\{0\}=\mathcal{B}_{0} \subset \cdots \mathcal{B}_{n}=\mathcal{C}$ is finite, $\mathcal{F}(\Delta)$ consists of finitely presented functors.

Proof. (i) Let $B \in \mathcal{C}$, then $B \in \mathcal{B}_{i}$ for some $i \in \mathbb{N}$. By Lemma 2.12 there exists an exact sequence $\mathcal{C}\left(-, E_{i-1}\right) \rightarrow \mathcal{C}\left(-, E_{i}\right) \rightarrow F^{(i)} \rightarrow 0$. If follows that $\operatorname{dim}_{K} F^{(i)}(B)<\infty$. Since $\left.\left.F\right|_{\mathcal{B}_{i}} \cong F^{(i)}\right|_{\mathcal{B}_{i}}$ by (17), we have $F(B)=\left.F\right|_{\mathcal{B}_{i}}(B)=$ $F^{(i)}(B)$, i.e, $\operatorname{dim}_{K} F(B)<\infty$.

(ii) Assume that there exists an exact sequence

$$
\mathcal{C}\left(-, C^{\prime}\right) \rightarrow \mathcal{C}\left(-, C^{\prime}\right) \rightarrow F \rightarrow 0
$$

with $C, C^{\prime} \in \mathcal{C}$. Then, there exists some $i \geq 0$ such that $C, C^{\prime} \in \mathcal{B}_{i}$. In this way, $F$ is projectively presented over $\mathcal{B}_{i}$ and $\left.\mathcal{C} \otimes_{\mathcal{B}_{i}} F\right|_{\mathcal{B}_{i}} \cong F$, but $F^{(i)} \cong \mathcal{C} \otimes_{\mathcal{B}_{i}}\left(\left.F\right|_{\mathcal{B}_{i}}\right)$ by part (i) of Lemma (2.12).

The other implication is a consequence of part (ii) of (2.12). (iii) If the filtration is finite: $0=\mathcal{B}_{0} \subset \mathcal{B}_{1} \subset \cdots \mathcal{B}_{n}=\mathcal{C}$, we have $F^{(n)}=F$, and the assertion follows from (ii).

Now we want to have a more specific characterization of $\mathcal{F}(\Delta)$ when the filtration $0=\mathcal{B}_{0} \subset \mathcal{B}_{1} \subset \cdots \mathcal{B}_{n}=\mathcal{C}$ is finite. For this we have the following lemma.

Assume that $\mathcal{C}$ is a quasi-hereditary with respect to a filtration $\left\{\mathcal{B}_{i}\right\}_{i \geq 0}$ which is not necessarialy finite. 
Lemma 2.14. Let $f: F \rightarrow G$ be an epimorphism of finitely presented $\mathcal{C}$-modules, for which there are projective presentations $\mathcal{C}\left(-, E_{i-1}\right) \rightarrow \mathcal{C}\left(-, E_{i}\right) \rightarrow F^{(i)} \rightarrow 0$, $\mathcal{C}\left(-, E_{i-1}^{\prime}\right) \rightarrow \mathcal{C}\left(-, E_{i}^{\prime}\right) \rightarrow G^{(i)} \rightarrow 0$, with $E_{i-1}, E_{i-1}^{\prime} \in \mathcal{B}_{i-1}$ and $E_{i}, E_{i}^{\prime} \in \mathcal{B}_{i}$, for all $i \geq 0$. Then, $f$ induces an epimorphism $f^{(i)}: F^{(i)} \rightarrow G^{(i)}$ and the following short exact sequence

$$
0 \rightarrow(\operatorname{Ker} f)^{(i)} /(\operatorname{Ker} f)^{(i-1)} \rightarrow F^{(i)} /(F)^{(i)} \rightarrow G^{(i)} / G^{(i-1)} \rightarrow 0
$$

for all $i \geq 1$.

Proof. The morphism $F^{(i)} \rightarrow G^{(i)}$ induced by $f$ is surjective because every map from $\mathcal{C}(-, E) \rightarrow G, E \in \mathcal{B}_{i}$, lifts to $F$. In this way, we have a commutative diagram

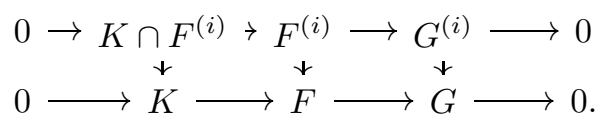

We show that $K^{(i)}=K \cap F^{(i)}$. Indeed let $E \in \mathcal{B}_{i}$ and consider any map $\psi$ : $\mathcal{C}(-, E) \rightarrow K$. Thus, the image of $\mathcal{C}(-, E) \stackrel{\psi}{\rightarrow} K \rightarrow F$ is contained in $F$ and therefore in $F^{(i)}$. Thus $K^{(i)} \subset K \bigcap F^{(i)}$.

On the other hand, since $K \bigcap F^{(i)} \subset K$, we have $\left(K \bigcap F^{(i)}\right)^{(i)} \subset K^{(i)}$. It is enough to prove $\left(K \cap F^{(i)}\right)^{(i)}=K \bigcap F^{(i)}$. Consider the following minimal projective presentations

$$
\begin{aligned}
\mathcal{C}\left(-, E_{i-1}\right) & \rightarrow \mathcal{C}\left(-, E_{i}\right) \rightarrow F^{(i)} \rightarrow 0, \\
\mathcal{C}\left(-, E_{i-1}^{\prime}\right) & \rightarrow \mathcal{C}\left(-, E_{i}^{\prime}\right) \rightarrow G^{(i)} \rightarrow 0,
\end{aligned}
$$

with $E_{i-1}, E_{i-1}^{\prime} \in \mathcal{B}_{i-1}$ and $E_{i}, E_{i}^{\prime} \in \mathcal{B}_{i}$. Thus, we have the following commutative diagram:

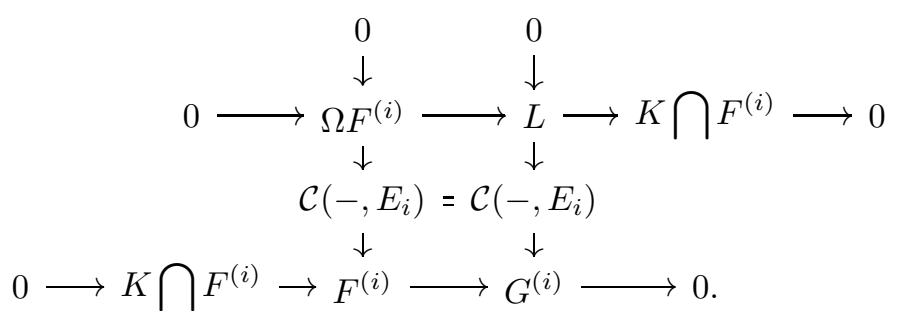

Thus, $\mathcal{C}\left(-, E_{i}^{\prime}\right)$ is a direct summand of $\mathcal{C}\left(-, E_{i}\right)$, i.e, $\mathcal{C}\left(-, E_{i}\right) \cong \mathcal{C}\left(-, E_{i}^{\prime}\right) \coprod Q$ for some $\mathcal{C}$-module $Q$, and as a consequence $L \cong Q \coprod \Omega G^{(i)}$. In this way $L$ can be covered by $\mathcal{C}\left(-, E_{i}\right) \coprod \mathcal{C}\left(-, E_{i-1}^{\prime}\right)$. Thus, there is an epimorphism

$$
\mathcal{C}\left(-, E_{i}\right) \coprod \mathcal{C}\left(-, E_{i-1}^{\prime}\right) \rightarrow K \bigcap F^{(i)},
$$

and therefore $\left(K \bigcap F^{(i)}\right)^{(i)}=K \bigcap F^{(i)}$.

Assume $\mathcal{C}$ is Noetherian and $F$ is a finitely presented functor. Thus, the subfunctor $F^{(i)} \subset F$ is finitely generated, and therefore finitely presented. In this way, the existence of a epimorphism $\coprod_{E \in \mathcal{B}_{i}}\left(-, B_{i}\right) \rightarrow F^{(i)}$ implies the existence of a epimorphism $(-, B) \rightarrow F^{(i)}$ with $B \in \mathcal{B}_{i}$; morever $F^{(i)}$ has a presentation as that required in the conditions of Lemma 2.14. On the other hand if $F$ is in $\mathcal{F}(\Delta)$ we have the same presentation by Lemma 2.12 .

In the next results, we have more explicit characterizations of $\mathcal{F}(\Delta)$ when $0=$ $\mathcal{B}_{0} \subset \mathcal{B}_{1} \subset \cdots \mathcal{B}_{n}=\mathcal{C}$ is a finite filtration. 
Theorem 2.15. $\quad$ (i) Assume that $\mathcal{C}$ has a finite filtration $0=\mathcal{B}_{0} \subset \mathcal{B}_{1} \subset$ $\cdots \mathcal{B}_{n}=\mathcal{C}$. Let $F \in \mathcal{F}(\Delta)$, then $\operatorname{Ext}^{1}(F, \nabla)=\operatorname{Tor}_{1}\left(F, \Delta^{\circ}\right)=0$.

(ii) $\mathcal{F}(\Delta)$ is closed under kernels of epimorphisms. In adition, if $0=\mathcal{B}_{0} \subset$ $\mathcal{B}_{1} \subset \cdots \mathcal{B}_{n}=\mathcal{C}$ is finite we have

$$
\operatorname{Ext}^{t}(F, \nabla)=\operatorname{Tor}_{t}\left(F, \Delta^{\circ}\right)=0, \text { for all } F \in \mathcal{F}(\Delta) \text { and } t \geq 1 \text {. }
$$

Proof. (i) It can be proved by induction that $\operatorname{Ext}^{1}\left(F^{(i)}, \nabla\right)=0$. The case $i=1$ is trivial since $F^{(1)}$ is projective. Assume that $\operatorname{Ext}^{1}\left(F^{(i-1)}, \nabla\right)=0$; hence, after applying $\operatorname{Ext}^{1}(-, \nabla)$ to the exact sequence $0 \rightarrow F^{(i-1)} \rightarrow F^{(i)} \rightarrow F^{(i)} / F^{(i)} \rightarrow 0$ we have $\operatorname{Ext}^{1}\left(F^{(i)}, \nabla\right)=0$ by Lemma 2.10.

(ii) Let $0 \rightarrow \operatorname{Ker} f \rightarrow F \stackrel{f}{\rightarrow} G \rightarrow 0$ be an epimorphism with $F, G \in \mathcal{F}(\Delta)$. For each $i \geq 1$, Lemma 2.14 gives the (split) exact sequence (18) of projective $\mathcal{C} / I_{\mathcal{B}_{i}}$ modules. Hence, $(\operatorname{Ker} f)^{(i)} /(\operatorname{Ker} f)^{(i-1)}$ is a direct summand of $F^{(i)} / F^{(i-1)}$. Given $F \in \mathcal{F}(\Delta)$ and an exact sequence $0 \rightarrow F^{\prime} \rightarrow \mathcal{C}(-, C) \rightarrow F \rightarrow 0$, we have $F^{\prime} \in \mathcal{F}(\Delta)$ because $\mathcal{C}(-, C) \in \mathcal{F}(\Delta)$ since $\mathcal{C}$ is quasi-hereditary. Therefore, $\operatorname{Ext}^{t+1}(F, \nabla)=$ $\operatorname{Ext}^{t}\left(F^{\prime}, \nabla\right)=0$ for all $t \geq 1$, and the proof follows by induction and part (i).

To obtain a result similar to $\bmod (\Lambda)$ for finite-dimensional algebras $\Lambda$, we add the condition that $\mathcal{C}$ is Noetherian; in this way if we take $F \in \bmod (\mathcal{C})$, then $F^{(i)} \in \bmod (\mathcal{C})$ for every $i \geq 1$.

Now we give one of the main results of this section (see Proposition A.2.3 in Dlab).

Theorem 2.16. Assume $\mathcal{C}$ is Noetherian and $(\mathcal{B})$ is a finite filtration for $\mathcal{C}$. Then,

$$
\mathcal{F}(\Delta)=\left\{F \mid \operatorname{Tor}_{1}\left(F, \Delta^{\circ}\right)=0\right\} .
$$

Proof. By Lemma $\mathcal{F}(\Delta) \subset\left\{F \mid \operatorname{Tor}_{1}\left(F, \Delta^{\circ}\right)=0\right\}$.

Now we will prove by reverse induction that

$$
\left\{F \mid \operatorname{Tor}_{1}\left(F, \Delta^{\circ}\right)=0 \text { and } F^{(j)}=0\right\} \subset \mathcal{F}(\Delta),
$$

for $j=1, \ldots, n$.

The case $j=n$ is trivial. Assume that the statement is true for $j=i$. Let $F \in\left\{X \mid \operatorname{Tor}_{1}\left(X, \Delta^{\circ}\right)=0\right.$ and $\left.X^{(i-1)}=0\right\}$. We have the following exact sequences

$$
\begin{array}{r}
0 \rightarrow F^{(i)} \rightarrow F \rightarrow G \rightarrow 0, \quad G^{(i)}=0, \\
0 \rightarrow H \rightarrow \coprod \Delta_{E^{\prime}}(i) \rightarrow F^{(i)} \rightarrow 0,
\end{array}
$$

where $\amalg \Delta_{E^{\prime}}(i)$ is a finite coproduct of $\mathcal{C}$-modules $\Delta_{E^{\prime}}(i)=\mathcal{C}\left(-, E^{\prime}\right) / I_{\mathcal{B}_{i-1}}\left(-, E^{\prime}\right)$, with $E^{\prime} \in \mathcal{B}_{i}$.

a) First, we show the existence of the exact sequence (20).

Let $\mathcal{C}\left(-, E_{i}\right) \stackrel{\varphi}{\rightarrow} F^{(i)} \rightarrow 0, E_{i} \in \mathcal{B}_{i}$, be a projective cover. Thus, we have a commutative diagram

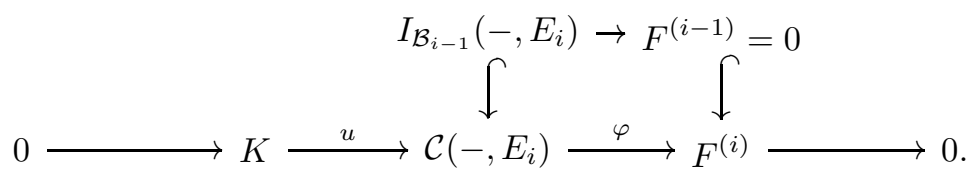


In this way, we have a commutative diagram

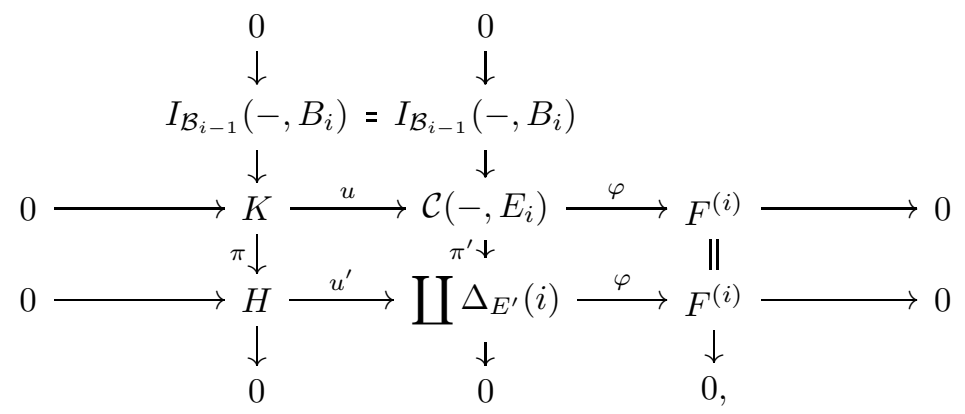

where $\coprod \Delta_{E^{\prime}}(i)$ is a finite sum, $E^{\prime} \in \mathcal{B}_{i}$.

It is clear that $H^{(i-1)}=\left(\frac{K}{I_{\mathcal{B}_{i-1}}\left(-, E_{i}\right)}\right)^{(i-1)}=0$. Now we show $H^{(i)}=0$. Indeed we put $E_{i}=\coprod_{t=1}^{s} E_{t}^{\prime}$ as a sum of indescomposable sumands. Let $\psi: \mathcal{C}(-, \tilde{E}) \rightarrow H$ any morphism, with $\tilde{E} \in \mathcal{B}_{i}$ an indecomposable object. Since $\mathcal{C}(-, \tilde{E})$ is projective, there exists a map $p: \mathcal{C}(-, \tilde{E}) \rightarrow K$ such that $\pi p=\psi$. Let $p_{i}: \coprod_{t=1}^{s} E_{t}^{\prime} \rightarrow E_{i}^{\prime}$ be a projection. It follows that the composition

$$
\mathcal{C}(-, \tilde{E}) \stackrel{p}{\rightarrow} K \stackrel{u}{\rightarrow} \mathcal{C}\left(-, \coprod_{t=1}^{s} E_{t}^{\prime}\right) \stackrel{p_{i}}{\rightarrow} \mathcal{C}\left(-, E_{i}^{\prime}\right)
$$

is an isomorphism or it lies in $\operatorname{rad} \mathcal{C}\left(\tilde{E}, E_{i}^{\prime}\right)=I_{\mathcal{B}_{i-1}}\left(\tilde{E}, E_{i}^{\prime}\right)$. Assume the above morphism is an isomorphism, then we get a contradiction with the fact that $\varphi$ is a projective cover. Therefore, the above morphism lies in $I_{\mathcal{B}_{i-1}}\left(\tilde{E}, E_{i}^{\prime}\right)$, and we get $q u p=u^{\prime} \psi=0$; finally $\psi=0$ because $u^{\prime}$ is a monomorphism.

b) Assume that $i<j$, then $F^{(i)} \otimes \Delta^{\circ}(j)=0$. Indeed since $i<j$, we have $i \leq j-1$ and $\mathcal{B}_{i} \subset \mathcal{B}_{j-1}$. Set $\Delta_{E}^{\circ}(j)=\frac{\mathcal{C}(E,-)}{I_{\mathcal{B}_{j-1}}(E,-)} \in \Delta^{\circ}(j)$, then there exists an epimorphism $\mathcal{C}\left(-, E_{i}\right) \stackrel{\varphi}{\rightarrow} F^{(i)} \rightarrow 0$, with $E_{i} \in \mathcal{B}_{i}$, which implies the epimorphism

$$
0=\frac{\mathcal{C}\left(E, E_{i}\right)}{I_{\mathcal{B}_{j-1}}\left(E, E_{i}\right)}=\mathcal{C}\left(-, E_{i}\right) \otimes \Delta_{E}^{\circ}(j) \rightarrow F^{(i)} \otimes \Delta_{E}^{\circ}(j) \rightarrow 0,
$$

since $E \in \mathcal{B}_{j}$.

c) Assume that $j \leq i$, then $\operatorname{Tor}_{1}(G, \Delta(j))=0$. Set $\Delta_{E}(j)=\frac{\mathcal{C}(E,-)}{I_{\mathcal{B}_{j-1}}(E,-)}$. Thus, there exist exact sequences

$$
\begin{array}{r}
\mathcal{C}\left(E_{j-1},-\right) \rightarrow I_{\mathcal{B}_{j-1}}(E,-) \rightarrow 0, E_{j-1} \in \mathcal{B}_{j-1}, \\
0 \rightarrow I_{\mathcal{B}_{j-1}}(E,-) \rightarrow \mathcal{C}(E,-) \rightarrow \Delta_{E}^{\circ}(j) \rightarrow 0 .
\end{array}
$$

On the other hand, the fact $j \leq i$ implies $\mathcal{B}_{j-1} \leq \mathcal{B}_{i-1}$ and $G^{(j-1)} \subset G^{(i-1)} \subset$ $G(i)=0$. Therefore $G \otimes \mathcal{C}\left(E_{j-1},-\right)=G\left(E_{j-1}\right)=\left(\mathcal{C}\left(-, E_{j-1}\right), G\right)=0$ because $G^{(j-1)}=0$. Thus, the exact sequence (21) implies the following exact sequence: $0=G \otimes \mathcal{C}\left(E_{j-1},-\right) \rightarrow G \otimes I_{\mathcal{B}_{j-1}}(E,-) \rightarrow 0$. Thus,

$$
G \otimes I_{\mathcal{B}_{j-1}}(E,-)=0 .
$$

After applying $G \otimes-$ to the exact sequence (22) and by using (23), we have the following exact sequence:

$$
0 \rightarrow \operatorname{Tor}_{1}\left(G, \Delta_{E}^{\circ}(j)\right) \rightarrow G \otimes I_{\mathcal{B}_{j-1}}(E,-) \rightarrow G \otimes \mathcal{C}(E,-) \rightarrow G \otimes \Delta_{E}^{\circ}(j) \rightarrow 0,
$$

which implies $\operatorname{Tor}_{1}\left(G, \Delta_{E}^{\circ}(j)\right)=0$, by (23) . 
d) $\operatorname{Tor}_{1}\left(G, \Delta^{\circ}(j)\right)=0$ for all $j \geq 0$. First, observe that after applying $-\otimes \Delta^{\circ}(j)$ to the exact sequence (19) and by using the fact $\operatorname{Tor}_{1}\left(F, \Delta^{\circ}(j)\right)=0$, we obtain by the long homology sequence the following exact sequence

$$
\begin{array}{r}
(24) \cdots \rightarrow \operatorname{Tor}_{2}\left(G, \Delta^{\circ}(j)\right) \rightarrow \operatorname{Tor}_{1}\left(F^{(i)}, \Delta^{\circ}(j)\right) \rightarrow 0 \rightarrow \operatorname{Tor}_{1}\left(G, \Delta^{\circ}(j)\right) \rightarrow \\
F^{(i)} \otimes \Delta^{\circ}(j) \rightarrow F \otimes \Delta^{\circ}(j) \rightarrow G \otimes \Delta^{\circ}(j) \rightarrow 0,
\end{array}
$$

for all $j \geq 0$.

By part (c), it only remains to prove $\operatorname{Tor}_{1}\left(G, \Delta^{\circ}(j)\right)=0$ when $i<j$. By part (b), however, we have $G^{(i)} \otimes \Delta^{\circ}(j)=0$, and therefore we get $\operatorname{Tor}_{1}\left(G, \Delta^{\circ}(j)\right)=0$ by the sequence (24).

e) $G \in F(\Delta)$ and $\operatorname{Tor}_{1}\left(G, \Delta^{\circ}\right)=\operatorname{Tor}_{1}\left(F^{(i)}, \Delta^{\circ}\right)=0$. In part (d), we proved $\operatorname{Tor}_{1}\left(G, \Delta^{\circ}(j)\right)=0$, and since $G^{(i)}=0$ it follows that $G \in F(\Delta)$ by induction hypothesis. Thus, $\operatorname{Tor}_{2}\left(G, \Delta^{\circ}\right)=0$ by Lemma 2.15, Finally, it follows from (24) that $\operatorname{Tor}_{1}\left(F^{(i)}, \Delta^{\circ}\right)=0$.

f) $H \otimes \Delta^{\circ}(j)=0$ for all $j \geq 0$. The exact sequence (20) implies the following long exact sequence:

$$
(25) \operatorname{Tor}_{1}\left(F^{(i)}, \Delta^{\circ}(j)\right) \rightarrow H \otimes \Delta^{\circ}(j) \rightarrow \Delta(i) \otimes \coprod \Delta_{E^{\prime}}^{\circ}(j) \rightarrow F^{(i)} \otimes \Delta^{\circ}(j) \rightarrow 0 .
$$

First assume that $i \neq j$. Then, $\Delta(i) \otimes \Delta^{\circ}(j)=0$ by Lemma 2.10 therefore the exact sequence (25) and part (e) imply that $H \otimes \Delta^{\circ}(j)=0$.

Assume $j=i$, then there exist exact sequences

$$
\mathcal{C}\left(E^{\prime},-\right) \rightarrow \Delta_{E^{\prime}}^{\circ}(i) \rightarrow 0, E^{\prime} \in \mathcal{B}_{i}
$$

which implies $H \otimes \mathcal{C}\left(E^{\prime},-\right) \rightarrow H \otimes \Delta_{E^{\prime}}^{\circ}(i) \rightarrow 0$. Since $H^{(i)}=0$, we have $H \otimes$ $\mathcal{C}\left(E^{\prime},-\right)=H\left(E^{\prime}\right)=\left(\mathcal{C}\left(-, E^{\prime}\right), H\right)=0$. It follows that $H \otimes \Delta^{\circ}(i)=0$.

g) $H \cong 0$. By induction, let $E_{1} \in \mathcal{B}_{1}$ and put $\Delta_{E_{1}}^{\circ}(1)=\frac{\mathcal{C}\left(E_{1},-\right)}{I_{\mathcal{B}_{0}}\left(E_{1},-\right)}=\mathcal{C}\left(E_{1},-\right)$. It follows that $H\left(E_{1}\right)=H \otimes \mathcal{C}\left(E_{1},-\right)=H \otimes \Delta_{E_{1}}^{\circ}(1)=0$. Therefore $H\left(E_{1}\right)=0$ for all $E_{1} \in \mathcal{B}_{1}$.

Assume $H\left(E_{i-1}\right)=0$ for all $E_{i-1} \in \mathcal{B}_{i-1}$. Let $E_{i} \in \mathcal{B}_{i}$, then $H \otimes I_{\mathcal{B}_{i-1}}\left(E_{i},-\right)=$ 0 . Indeed we have an exact sequence

(26) $\mathcal{C}\left(E_{i-2},-\right) \rightarrow \mathcal{C}\left(E_{i-1},-\right) \rightarrow I_{\mathcal{B}_{i-1}}\left(E_{i},-\right) \rightarrow 0, E_{i-2} \in \mathcal{B}_{i-2}, E_{i-1} \in \mathcal{B}_{i-1}$.

which implies

$$
0=H\left(E_{i-1}\right)=H \otimes \mathcal{C}\left(E_{i-1},-\right) \rightarrow H \otimes I_{\mathcal{B}_{i-1}}\left(E_{i},-\right) \rightarrow 0 .
$$

As a result $H \otimes I_{\mathcal{B}_{i-1}}\left(E_{i},-\right)=0$.

In this way, there exists an exact sequence

$$
0 \rightarrow I_{\mathcal{B}_{i-1}}\left(E_{1},-\right) \rightarrow \mathcal{C}\left(E_{1},-\right) \rightarrow \frac{\mathcal{C}\left(E_{i},-\right)}{I_{\mathcal{B}_{i-1}}\left(E_{i},-\right)}=\Delta_{E_{i}}^{\circ}(i) \rightarrow 0,
$$

which implies

$$
\begin{array}{r}
H \otimes I_{\mathcal{B}_{i-1}}\left(E_{1},-\right) \rightarrow H \otimes \mathcal{C}\left(E_{i},-\right) \longrightarrow H \otimes \Delta_{E_{i}}^{\circ}(i) \longrightarrow \\
\stackrel{\downarrow}{\cong \downarrow} \\
0 \longrightarrow \\
0
\end{array}
$$

by part (f). Therefore $H\left(E_{i}\right)=0$ for all $E_{i} \in \mathcal{B}_{i}$.

h) Since $H \cong 0$ and $G \in \mathcal{F}(\Delta)$, the sequences (19) and (20) imply that $F \in$ $\mathcal{F}(\Delta)$. 
2.5. Quasi-hereditary categories and their subcategories. Assume that $\mathcal{C}$ is a Hom-finite Krull-Schmidt quasi-hereditary category $K$-category with an exhaustive filtration $\left\{\mathcal{B}_{i}\right\}_{i \geq 0}$ :

$$
0=\mathcal{B}_{0} \subset \cdots \subset \mathcal{B}_{i-1} \subset \mathcal{B}_{i} \subset \cdots \subset \mathcal{C} .
$$

According with the definition, each $\mathcal{B}_{i}$ is a quasi-hereditary category. We denote by

$$
\Delta_{\mathcal{B}_{i}}=\{\hat{\Delta}(j) \mid 0 \leq j \leq i\},
$$

the corresponding sequence of standard subcategories with respect to the filtration

$$
0=\mathcal{B}_{0} \subset \cdots \subset \mathcal{B}_{i-1} \subset \mathcal{B}_{i}
$$

where

and

$$
\hat{\Delta}(j)=\left\{\mathcal{B}_{i}(-, E) / \hat{I}_{\mathcal{B}_{j-1}}(-, E) \mid E \in \mathcal{B}_{j}\right\}
$$

$$
\hat{I}_{\mathcal{B}_{j-1}}(-, E)=\operatorname{Tr}_{\left\{\mathcal{B}_{i}\left(-, E^{\prime}\right) \mid E^{\prime} \in \mathcal{B}_{j-1}\right\}} \mathcal{B}_{i}(-, E)
$$

Thus, there is an associated category $\mathcal{F}\left(\Delta_{\mathcal{B}_{i}}\right) \subset \bmod \left(\mathcal{B}_{i}\right)$ consisting of $\mathcal{B}_{i^{-}}$ modules which have a $\Delta_{\mathcal{B}_{i}}$-filtration.

Here we study a relationship between $\mathcal{F}\left(\Delta_{\mathcal{B}_{i}}\right)$ and $\mathcal{F}\left(\Delta_{\mathcal{C}}\right)$. Remember that there is a pair of functors

$$
\left.\right|_{\mathcal{B}_{i}}: \bmod (\mathcal{C}) \rightarrow \bmod \left(\mathcal{B}_{i}\right), \mathcal{C} \otimes_{\mathcal{B}_{i}}-: \bmod \left(\mathcal{B}_{i}\right) \rightarrow \bmod (\mathcal{C}) .
$$

We denote by $\bmod (\mathcal{C})^{(i)}$ the full subcategory of all $F \in \mathcal{F}\left(\Delta_{\mathcal{C}}\right)$ for which $F=F^{(i)}$

Theorem 2.17. Let $\mathcal{C}$ be a quasi-hereditary category with respect to $\left\{\mathcal{B}_{i}\right\}_{i \geq 0}$. The restrictions of the functors (27) define an equivalence of $\bmod (\mathcal{C})^{(i)}$ and $\mathcal{F}\left(\Delta_{\mathcal{B}_{i}}\right)$

Proof. 1) Let $G \in \mathcal{F}\left(\Delta_{\mathcal{B}_{i}}\right)$ with filtration

$$
G^{(0)} \subset G^{(1)} \subset \cdots \subset G^{(i-1)} \subset G^{(i)}=G .
$$

We will show that $\mathcal{C} \otimes_{\mathcal{B}_{i}} G$ lies in $\bmod (\mathcal{C})^{(i)}$.

Consider the following exact sequence

$$
\mathcal{B}_{i}\left(-, E^{\prime}\right) \rightarrow \mathcal{B}_{i}(-, E) \rightarrow G \rightarrow 0,
$$

with $E^{\prime}, E \in \mathcal{B}_{i}$. Thus, after applying $\mathcal{C} \otimes_{\mathcal{B}_{i}}$ to (28), we obtain

$$
\mathcal{C}\left(-E^{\prime}\right) \rightarrow \mathcal{C}(-, E) \rightarrow \mathcal{C} \otimes_{\mathcal{B}_{i}} G \rightarrow 0
$$

a) First we prove that

$$
\left(\mathcal{C} \otimes_{\mathcal{B}_{i}} G\right)^{(j)}= \begin{cases}\mathcal{C} \otimes_{\mathcal{B}_{i}} G & \text { if } j<i \\ \mathcal{C} \otimes_{\mathcal{B}_{i}} G & \text { if } j \geq i .\end{cases}
$$

a.1) Assume that $j<i$. Given $E \in B_{i}$, there is a pair of functors

$$
\begin{array}{r}
I_{\mathcal{B}_{j}}(-, E) \subset \mathcal{C}(-, E): \bmod (\mathcal{C}) \rightarrow \mathbf{A b}, \\
\hat{I}_{\mathcal{B}_{j}}(-, E) \subset \mathcal{B}_{i}(-, E): \bmod \left(\mathcal{B}_{i}\right) \rightarrow \mathbf{A b} .
\end{array}
$$

Clearly, we have

$$
\left.I_{\mathcal{B}_{j}}(-, E)\right|_{\mathcal{B}_{i}}=\hat{I}_{\mathcal{B}_{j}}(-, E) .
$$


Now consider the exact sequence

$$
\mathcal{C}\left(-, E_{j-1}\right) \rightarrow \mathcal{C}\left(-, E_{j}\right) \rightarrow I_{\mathcal{B}_{j}}(-, E) \rightarrow 0,
$$

with $E_{j} \in \mathcal{B}_{j}, E_{j-1} \in \mathcal{B}_{j-1}$. Since $j<i$, we have $E_{j}, E_{j-1} \in B_{i}$. In this way, $I_{\mathcal{B}_{j}}(-, E)$ is projectively presented over $B_{i}$; thus we have

$$
\begin{aligned}
I_{\mathcal{B}_{j}}(-, E) & =\mathcal{C} \otimes_{\mathcal{B}_{i}}\left(\left.I_{\mathcal{B}_{j}}(-, E)\right|_{\mathcal{B}_{i}}\right) \\
& =\mathcal{C} \otimes_{\mathcal{B}_{i}} \hat{I}_{\mathcal{B}_{j}}(-, E) .
\end{aligned}
$$

After applying $\left.\right|_{\mathcal{B}_{j}}$ followed by $\mathcal{B}_{i} \otimes_{\mathcal{B}_{j}}$ to the exact sequence (28), we obtain by Lemma 2.12 the following exact sequence:

$$
\hat{I}_{\mathcal{B}_{j}}\left(-, E^{\prime}\right) \rightarrow \hat{I}_{\mathcal{B}_{j}}(-, E) \rightarrow G^{(j)} \rightarrow 0 .
$$

After applying $\mathcal{C} \otimes_{\mathcal{B}_{i}}$ to the exact sequence (31) and by using (30) we have the following exact sequence

$$
I_{\mathcal{B}_{j}}(-, E) \rightarrow I_{\mathcal{B}_{j}}(-, E) \rightarrow \mathcal{C} \otimes_{\mathcal{B}_{i}} G^{(j)} \rightarrow 0
$$

After applying $\left.\right|_{\mathcal{B}_{j}}$ followed by $\mathcal{C} \otimes_{\mathcal{B}_{j}}$ to the exact sequence (29) and by using Lemma 2.12, we obtain the following exact sequence

$$
I_{\mathcal{B}_{j}}\left(-, E^{\prime}\right) \rightarrow I_{\mathcal{B}_{j}}(-, E) \rightarrow\left(\mathcal{C} \otimes_{\mathcal{B}_{i}} G\right)^{(j)} \rightarrow 0 .
$$

Therefore, we obtain

$$
\mathcal{C} \otimes_{\mathcal{B}_{i}} G^{(j)} \cong\left(\mathcal{C} \otimes_{\mathcal{B}_{i}} G\right)^{(j)}
$$

by (33) and (32).

a.2) Assume $j \geq i$. Thus, after applying $\left.\right|_{\mathcal{B}_{j}}$ followed by $\mathcal{C} \otimes_{\mathcal{B}_{j}}$ to the exact sequence (29), we have

$$
I_{\mathcal{B}_{j}}\left(-E^{\prime}\right) \rightarrow I_{\mathcal{B}_{j}}(-, E) \rightarrow\left(\mathcal{C} \otimes_{\mathcal{B}_{i}} G\right)^{(j)} \rightarrow 0 .
$$

Since $E^{\prime}, E \in \mathcal{B}_{i} \subset \mathcal{B}_{j}$, we conclude that $I_{\mathcal{B}_{j}}\left(-E^{\prime}\right)=\mathcal{C}\left(-, E^{\prime}\right)$ and $I_{\mathcal{B}_{j}}(-E)=$ $\mathcal{C}(-, E)$. Thus (34) can be written as

$$
\mathcal{C}\left(-E^{\prime}\right) \rightarrow \mathcal{C}(-, E) \rightarrow\left(\mathcal{C} \otimes_{\mathcal{B}_{i}} G\right)^{(j)} \rightarrow 0 .
$$

This implies that $\left(\mathcal{C} \otimes_{\mathcal{B}_{i}} G\right)^{(j)}=\mathcal{C} \otimes_{\mathcal{B}_{i}} G$ by (29).

b) Let $E \in \mathcal{B}_{j}$, and assume that $\hat{\Delta}_{E}(j)=\mathcal{B}_{i}(-, E) / \hat{I}_{\mathcal{B}_{j-1}}(-, E)$ is in $\hat{\Delta}(j)$. Then $\mathcal{C} \otimes \hat{\Delta}_{E}(j)$ lies in $\Delta(j)$. Indeed, consider the exact sequence

$$
0 \rightarrow \hat{I}_{\mathcal{B}_{j-1}}(-, E) \rightarrow \mathcal{B}_{i}(-, E) \rightarrow \hat{\Delta}_{E}(j) \rightarrow 0 .
$$

Then, after applying $\mathcal{C} \otimes_{\mathcal{B}_{i}}$ and using (30), we have a commutative diagram with exact arrows:

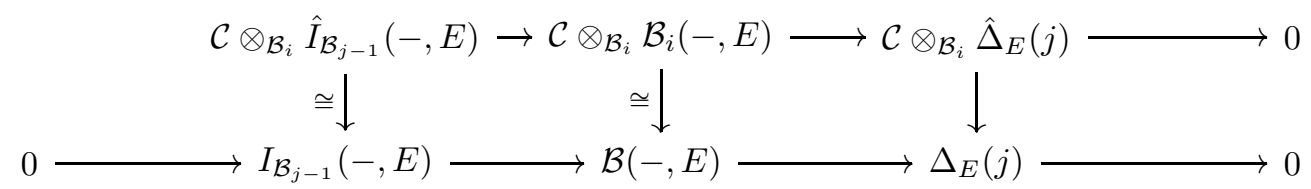

Therefore $\mathcal{C} \otimes \hat{\Delta}_{E}(j) \cong \Delta_{E}(j) \in \Delta(j)$.

c) We have a $\Delta_{\mathcal{C}}$-filtration

$$
(\mathcal{C} \otimes G)^{(0)} \subset(\mathcal{C} \otimes G)^{(1)} \subset(\mathcal{C} \otimes G)^{(2)} \subset \cdots
$$

such that $(\mathcal{C} \otimes G)^{(j)}=\mathcal{C} \otimes G$ for $j \geq i$. Indeed after applying $\mathcal{C} \otimes \mathcal{B}_{i}$ to the exact sequence

$$
0 \rightarrow G^{(j-1)} \rightarrow G^{(j)} \rightarrow \frac{G^{(j)}}{G^{(j-1)}} \rightarrow 0
$$


we obtain the following commutative diagram with exact arrows:

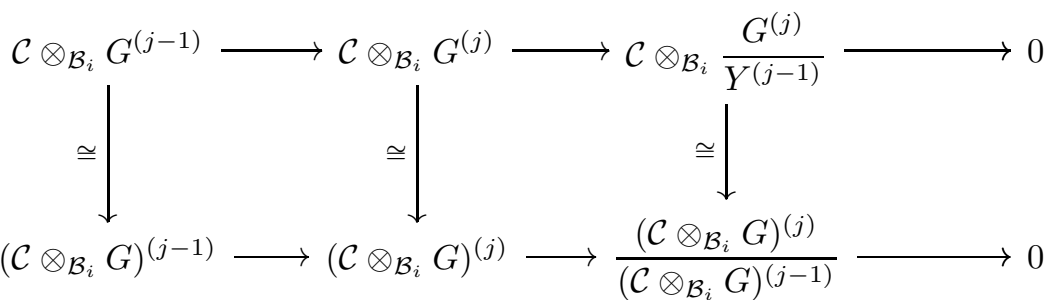

Thus, the map $\mathcal{C} \otimes_{\mathcal{B}_{i}} G^{(j-1)} \rightarrow \mathcal{C} \otimes_{\mathcal{B}_{i}} G^{(j)}$ is a monomorphism. Morever, we have

$$
\frac{\mathcal{C} \otimes_{\mathcal{B}_{i}} G^{(j)}}{\mathcal{C} \otimes_{\mathcal{B}_{i}} G^{(j-1)}} \cong \frac{\left(\mathcal{C} \otimes_{\mathcal{B}_{i}} G\right)^{(j)}}{\left(\mathcal{C} \otimes_{\mathcal{B}_{i}} G\right)^{(j-1)}} \cong \mathcal{C} \otimes_{\mathcal{B}_{i}}\left(\frac{G^{(j)}}{G^{(j-1)}}\right) .
$$

Since $\frac{G^{(j)}}{G^{(j-1)}}$ is a direct sum of objects from the category $\hat{\Delta}(j)$, we conclude that $\mathcal{C} \otimes_{\mathcal{B}_{i}} \frac{G^{(j)}}{G^{(j-1)}}$ is a direct sum of objects from the category $\Delta(j)$ by the part (b).

2) Let $F \in \bmod (\mathcal{C})^{(i)}$, and assume that $F$ has a trace filtration

$$
0=F^{(0)} \subset F^{(1)} \subset \cdots \subset F^{(i-1)} \subset F^{(i)}=F
$$

Since the functor restriction $\mid \mathcal{B}_{i}$ is exact, we have a filtration for $\left.F\right|_{\mathcal{B}_{i}}$ :

$$
0=\left.\left.\left.\left.F^{(0)}\right|_{\mathcal{B}_{i}} \subset F^{(1)}\right|_{\mathcal{B}_{i}} \subset \cdots \subset F^{(i-1)}\right|_{\mathcal{B}_{i}} \subset F^{(i)}\right|_{\mathcal{B}_{i}}=\left.F\right|_{\mathcal{B}_{i}}
$$

We will prove that (36) is a $\Delta_{\mathcal{B}_{i}}$-filtration for $\left.F\right|_{\mathcal{B}_{i}}$.

a) Assume that $j \leq i$. First we prove that $\left.\left(\left.F\right|_{\mathcal{B}_{i}}\right)^{(j)} \cong F^{(j)}\right|_{\mathcal{B}_{i}}$.

By Lemma 2.12 the functor $F^{(i)}$, and therefore $F$ has a presentation

$$
\mathcal{C}\left(-, E_{i-1}\right) \rightarrow \mathcal{C}\left(-, E_{i}\right) \rightarrow F \rightarrow 0,
$$

$E_{i-1} \in \mathcal{B}_{i-1}, E_{i} \in \mathcal{B}_{i}$

After applying $\left.\right|_{\mathcal{B}_{i}}$ to the exact sequence (37), we obtain the following exact sequence:

$$
\left.\mathcal{B}_{i}\left(-, E_{i-1}\right) \rightarrow \mathcal{B}_{i}\left(-, E_{i}\right) \rightarrow F\right|_{\mathcal{B}_{i}} \rightarrow 0,
$$

with $E_{j-1} \in \mathcal{B}_{j-1}, E_{j} \in \mathcal{B}_{j}$.

After applying $\mathcal{C} \otimes_{\mathcal{B}_{j}}\left(-\left.\right|_{\mathcal{B}_{j}}\right)$ and $\mathcal{B}_{i} \otimes_{\mathcal{B}_{j}}\left(-\left.\right|_{\mathcal{B}_{j}}\right)$ to the exact sequences (37) and (38) respectively, we obtain the following exact sequences:

$$
\begin{array}{r}
I_{\mathcal{B}_{j}}\left(-, E_{i-1}\right) \rightarrow I_{\mathcal{B}_{j}}\left(-, E_{i}\right) \rightarrow F^{(j)} \rightarrow 0, \\
\hat{I}_{\mathcal{B}_{j}}\left(-, E_{i-1}\right) \rightarrow \hat{I}_{\mathcal{B}_{j}}\left(-, E_{i}\right) \rightarrow\left(\left.F\right|_{\mathcal{B}_{i}}\right)^{(j)} \rightarrow 0,
\end{array}
$$

by Lemma 2.12 ,

After applying $\left.\right|_{\mathcal{B}_{i}}$ to the exact sequence (39), we obtain the exact sequence

$$
\left.\hat{I}_{\mathcal{B}_{j}}\left(-, E_{i-1}\right) \rightarrow \hat{I}_{\mathcal{B}_{j}}\left(-, E_{i}\right) \rightarrow F^{(j)}\right|_{\mathcal{B}_{i}} \rightarrow 0 .
$$

It follows from (40) and (41) that $\left.\left(\left.F\right|_{\mathcal{B}_{i}}\right)^{(j)} \cong F^{(j)}\right|_{\mathcal{B}_{i}}$.

b) $\left(\left.F\right|_{\mathcal{B}_{i}}\right)^{(j)} /\left(\left.F\right|_{\mathcal{B}_{i}}\right)^{(j-1)}$ is a finite sum of objects from $\hat{\Delta}(j)$. 
Let $\Delta_{E}(j)=\mathcal{C}(-, E) / I_{\mathcal{B}_{j-1}}(-, E)$ with $E \in \mathcal{B}_{j}$. Thus, after applying $\left.\right|_{\mathcal{B}_{i}}$ to the exact sequence $0 \rightarrow I_{\mathcal{B}_{j-1}}(-, E) \rightarrow \mathcal{C}(-, E) \rightarrow \Delta_{E}(j) \rightarrow 0$, we obtain the following commutative diagram:

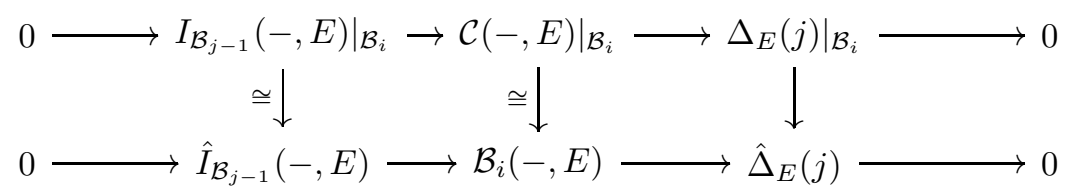

Thus, $\left.\Delta_{E}(j)\right|_{\mathcal{B}_{i}} \cong \hat{\Delta}_{E}(j)$.

Since $F^{(j)} / F^{(j-1)}$ is a finite sum of objects from $\Delta(j)$, it follows that the quotient

$$
\left.\frac{\left.F^{(j)}\right|_{\mathcal{B}_{i}}}{\left.F^{(j-1)}\right|_{\mathcal{B}_{i}}} \cong \frac{F^{(j)}}{F^{(j-1)}}\right|_{\mathcal{B}_{i}}
$$

is a finite sum of objects from $\hat{\Delta}(j)$.

\section{3. $\mathcal{F}(\Delta)$ IS FUNCTORIALLY FINITE}

In order to to have another analogous result closer to the theory of finitedimensional quasi-hereditary algebras, we add more restrictions to our categories; in particular, we need the existence of duality. We will assume in this section that $\mathcal{C}$ is a dualizing Krull-Schmidt $K$-variety. In this way, finitely presented functors have projective covers (see Theorem 2 in [MVO1]), and the category of finitely presented functors $\bmod (\mathcal{C})$ has enough projective and injective objects. In the rest of this work, all the $\mathcal{C}$-modules we are considering are finitely presented.

The main result of this section is to prove that $\mathcal{F}(\Delta)$ is functorially finite in $\bmod (\mathcal{C})$ if we add the additional condition that $\mathcal{C}$ is Noetherian Rin2. We begin the subsection recalling some definitions from $[\mathrm{AB}, \mathrm{AuRe}$.

Let $\mathcal{X}$ be a full subcategory of $\bmod (\mathcal{C})$; a morphism $f: X \rightarrow M$ in $\bmod (\mathcal{C})$, with $X$ in $\mathcal{X}$, is a right $\mathcal{X}$-approximation of $M$ if $(-, X)_{\mathcal{X}} \stackrel{(-, f)_{\mathcal{X}}}{\longrightarrow}(-, M)_{\mathcal{X}}$ is an exact sequence. Dually, let $\mathcal{Y}$ be a subcategory of $\bmod (\mathcal{C})$, a morphism $g: M \rightarrow Y$ is a left $\mathcal{Y}$-approximation of $M$ if $(Y,-) \mathcal{Y} \stackrel{(g,-) \mathcal{Y}}{\longrightarrow}(M,-) \mathcal{Y} \rightarrow 0$ is exact.

A subcategory $X$ of $\bmod (\mathcal{C})$ is contravariantly (covariantly) finite in $\mathcal{C}$ if every object $M$ in $\bmod (\mathcal{C})$ has a right (left) $\mathcal{X}$-approximation.

A subcategory $X$ of $\bmod (\mathcal{C})$ is resolving (coresolving) if it satisfies the following three conditions: (a) it is closed under extensions; (b) it is closed under kernels (cokerneles) of epimorphisms (monomorphisms) and (c) it contains the projective (injective) objects.

A full subcategory $\mathcal{X}$ in $\bmod (\mathcal{C})$ is said to be functorially finite in $\bmod (\mathcal{C})$ provided every $\mathcal{C}$-module has both a right $\mathcal{X}$-approximation and a left $\mathcal{X}$-approximation. Assume that $\mathcal{C}$ is quasi-hereditary with respect to a finite filtration $0=\mathcal{B}_{0} \subset \cdots \subset$ $\mathcal{B}_{n}=\mathcal{C}$. We follow closely the arguments given in Rin2, so we obtain the following

Theorem 3.1 ([Rin2], Theorem 1). Let $\mathcal{C}$ be a dualizing Krull-Schmidt Noetherian $K$-category. Assume $\mathcal{C}$ is quasi-hereditary with respect to a finite filtration $0=\mathcal{B}_{0} \subset$ $\cdots \subset \mathcal{B}_{n}=\mathcal{C}$, then $\mathcal{F}(\Delta)$ is functorially finite in $\bmod (\mathcal{C})$.

We start with an arbitrary subcategory $\mathcal{X}$ of $\bmod (\mathcal{C})$, and we denote by $\mathcal{Y}$ the full subcategory of $\bmod (\mathcal{C})$ of all modules $Y$ satisfying $\operatorname{Ext}_{\mathcal{C}}^{1}(X, Y)=0$. 
Lemma 3.2. Let $0 \rightarrow Y \rightarrow X \stackrel{\gamma}{\rightarrow} M \rightarrow 0$ be exact, with $X \in \mathcal{X}$ and $Y \in \mathcal{Y}$. Then $\gamma$ is a right $\mathcal{X}$-approximation of $M$.

Proof. This is the converse of Wakamatsu's lemma, and it is proved in [Ring].

Now let $\mathcal{X}=\mathcal{F}(\Delta)$. Then $\mathcal{Y}=\mathcal{Y}(\Delta)$ may be characterized alternatively as the full subcategory of $\mathcal{C}$-modules $\mathcal{Y}$ satisfiying $\operatorname{Ext}_{\mathcal{C}}^{1}(\Delta(i), Y)=0$ for $1 \leq i \leq n$.

In the rest of this section $\mathcal{C}$, will be a dualizing Krull-Schmidt Noetherian $K$ quasi-hereditary category with respect to a finite filtration $(\mathcal{B})$.

Lemma 3.3. Assume that $\mathcal{X}$ is closed under extensions and that for every $\mathcal{C}$-module $N$ there exists an exact sequence $0 \rightarrow N \rightarrow Y^{N} \rightarrow X^{N} \rightarrow 0$ with $X^{N} \in \mathcal{X}$ and $Y^{N} \in \mathcal{Y}$. Then every $\mathcal{C}$-module $M$ has a right $\mathcal{X}$-approximation.

Proof. a) Let $M$ be a finitely generated $\mathcal{C}$-module. Then, there is an epimorphism $\pi: X \rightarrow M$ with $X \in \mathcal{X}$. Let $M^{\prime}$ be the submodule of $M$ generated by the images of maps $X^{\prime} \rightarrow M$ with $X^{\prime} \in \mathcal{X}$. In this way, there exists an epimorphism $\coprod_{X \in \mathcal{F}(\Delta)} X \stackrel{\psi}{\rightarrow} M^{\prime}$. Since $M^{\prime}$ is a submodule of the finitely generated $\mathcal{C}$-module $M$, there exists an epimorphism $\varphi: \mathcal{C}(-, E) \rightarrow M^{\prime}$, since $\mathcal{C}$ is Noetherian. Therefore, there exists $i \geq 1$ such that $E \in \mathcal{B}_{i}$ and $\varphi$ lifts to $\psi$, in other words, the exists $f: \mathcal{C}(-, E) \rightarrow \coprod_{X \in \mathcal{F}(\Delta)} X$ such that $\psi f=\varphi$. Then, there is a finite family $\left\{X_{j}\right\}_{j=1}^{n}$ of objects in $\mathcal{F}(\Delta)$ for which the image of $f$ is contained in $\coprod_{j=1}^{n} X_{j}$. Thus, there exists an epimorphism $\coprod_{j=1}^{n} X_{j}^{(i)} \rightarrow M^{\prime}$. Since $\mathcal{F}(\Delta)$ is closed under direct sums, we can take $X=\coprod_{j=1}^{n} X_{j}^{(i)}$.

b) By part (a), there exists an exact sequence $0 \rightarrow K \rightarrow X \stackrel{\pi}{\rightarrow} M^{\prime} \rightarrow 0$ with $X \in \mathcal{X}:$

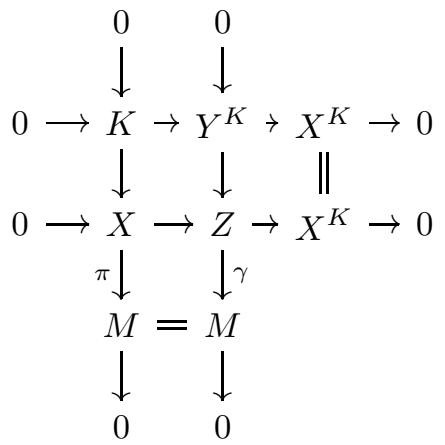

Since $X$ and $X^{K}$ belong to $\mathcal{X}$, and $\mathcal{X}$ is closed under extensions, $Z \in \mathcal{X}$. Since $Y^{K} \in \mathcal{Y}$, we use Lemma 3.2 for the exact sequence which appears as middle column and conclude that $\gamma: Z \rightarrow M^{\prime}$ is a right approximation. We denote by $\mu: M^{\prime} \rightarrow M$ the inclusion map. It is clear that $\mu \gamma$ is a right approximation.

Of course $\mathcal{X}=\mathcal{F}(\Delta)$ is closed under extensions and coproducts by Lemma 2.15 since $0=\mathcal{B}_{0} \subset \cdots \subset \mathcal{B}_{n}=\mathcal{C}$ is finite. Morever it contains the projective objects of $\bmod (\mathcal{C})$ because $\mathcal{C}$ is quasi-hereditary; thus, it is a coresolving category of $\bmod (\mathcal{C})$.

Lemma 3.4. Let $N \in \bmod (\mathcal{C})$ and $t \in\{1, \ldots, n\}$.

(i) Assume that $N$ is a finitely generated $\mathcal{C}$-module. Then $\operatorname{Ext}^{1}\left(\Delta_{E}(t), N\right)$ is finitely generated for all $E \in \mathcal{B}_{t}$.

(ii) Assume $N$ has finite length. Then, there is a finite number of $E$ 's in $\mathcal{B}_{t}$ for which $\operatorname{Ext}^{1}\left(\Delta_{E}(t), N\right) \neq 0$ (see [A3 Prop. 3.10]). 
Proof. i) Consider the folowing exact senquences:

$$
\begin{aligned}
& 0 \rightarrow I_{\mathcal{B}_{t}}(-, E) \rightarrow \mathcal{C}(-, E) \rightarrow \Delta_{E}(t) \rightarrow 0, \\
& \mathcal{C}\left(-, E_{t-1}\right) \rightarrow \mathcal{C}\left(-, E_{t}\right) \rightarrow I_{\mathcal{B}_{t}}(-, E) \rightarrow 0 .
\end{aligned}
$$

After applying $(-, N)$ to the above the exact sequences, we get the following exact sequences:

$$
\begin{array}{r}
0 \rightarrow\left(\Delta_{E}(t), N\right) \rightarrow(\mathcal{C}(-, E), N) \rightarrow\left(I_{\mathcal{B}_{t}}(-, E), N\right) \rightarrow \operatorname{Ext}^{1}\left(\Delta_{E}(t), N\right) \rightarrow 0 \\
0 \rightarrow\left(I_{\mathcal{B}_{t}}(-, E), N\right) \rightarrow\left(\mathcal{C}\left(-, E_{t}\right), N\right) \cong N\left(E_{t}\right) .
\end{array}
$$

Thus, $\left(I_{\mathcal{B}_{t}}(-, E), N\right)$ is finitely generated, and it follows that $\operatorname{Ext}^{1}\left(\Delta_{E}(t), N\right)$ is finitely generated.

ii) First, we prove that if $N$ is of finite length, there is then a finite number of $E$ 's in $\mathcal{B}_{t}$ for which $\operatorname{Hom}\left(\Delta_{E}(t), N\right) \neq 0$. By induction on the length $l(N)$ of $N$. If $l(N)=1, N$ is simple and the claim is true in this case. Assume that the fact is true for modules with lenght $<l(N)$, and consider the exact sequence $0 \rightarrow S \rightarrow N \rightarrow N / S \rightarrow 0$ which implies

$$
0 \rightarrow\left(\Delta_{E}(t), S\right) \rightarrow\left(\Delta_{E}(t), N\right) \rightarrow\left(\Delta_{E}(t), N / S\right)
$$

Then there is a finite number of $E$ 's in $\mathcal{B}_{t}$ for which $\left(\Delta_{E}(t), S\right) \neq 0$ and $\left(\Delta_{E}(t), N / S\right) \neq$ 0 ; therefore there is a finite number of $E$ 's in $\mathcal{B}_{t}$ for which $\left(\Delta_{E}(t), N\right) \neq 0$.

Now consider the exact sequence $0 \rightarrow N \rightarrow I \rightarrow \Omega^{-1} N \rightarrow 0$ where $I$ is the injective envelope of $N$. It implies

$$
0 \rightarrow\left(\Delta_{E}(t), N\right) \rightarrow\left(\Delta_{E}(t), N\right) \rightarrow\left(\Delta_{E}(t), \Omega^{-1} N\right) \rightarrow \operatorname{Ext}^{1}\left(\Delta_{E}(t), N\right) \rightarrow 0 .
$$

Since $\left(\Delta_{E}(t), \Omega^{-1} N\right) \neq 0$ only for a finite number of $E$ 's in $\mathcal{B}_{t}$, it follows that $\operatorname{Ext}^{1}\left(\Delta_{E}(t), N\right) \neq 0$ only for a finite number of $E$ 's in $\mathcal{B}_{t}$.

Lemma 3.5. Let $1 \leq t \leq n$. Let $N$ be a $\mathcal{C}$-module with $\operatorname{Ext}^{1}(\Delta(j), N)=0$ for all $j<t$. Then there exists an exact sequence $0 \rightarrow N \rightarrow N^{\prime} \rightarrow Q \rightarrow 0$ with $Q$ a direct sum of objects from $\Delta(t)$ and $\operatorname{Ext}^{1}\left(\Delta(j), N^{\prime}\right)=0$ for all $j \leq t$.

Let us fix an integer $t \geq 0$ and set $\Delta_{E}(t)=\mathcal{C}(-, E) / I_{\mathcal{B}_{t-1}}(-, E)$. By Lemma 3.4. the family

$$
B_{t}=\left\{E \in \mathcal{B}_{t} \mid \operatorname{Ext}\left(\Delta_{E}(t), N\right) \neq 0\right\}
$$

has a finite number of objects, say $m_{E}$. For all $E \in B_{t}$, let

$$
\left\{\xi_{E}^{i}: 0 \rightarrow N \rightarrow W_{E}^{i} \rightarrow \Delta_{E}(t) \rightarrow 0 \mid 1 \leq i \leq m_{E}\right\}
$$

be a $K$-basis for $\operatorname{Ext}^{1}\left(\Delta_{E}(t), N\right)$. Thus, we can take $\left\{\xi_{E}^{i} \mid 1 \leq i \leq m_{E}\right\}_{E \in B_{t}}$ as a $K$-basis for $\operatorname{Ext}^{1}\left(\coprod_{E \in B_{t}} \Delta_{E}(t), N\right)$.

In this way, we have a push-out diagram:

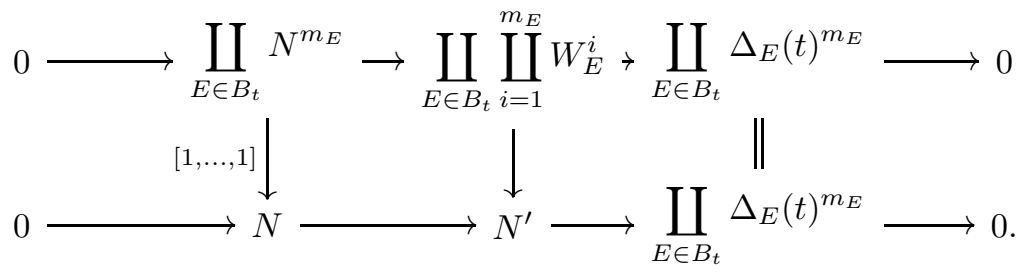


Let $u_{E}^{i}{ }^{\prime \prime}: N \rightarrow \coprod_{E \in B_{t}} N^{m_{E}}, u_{E}^{i}{ }^{\prime}: W_{E}^{i} \rightarrow \coprod_{E \in B_{t}} \coprod_{i=1}^{m_{E}} W_{E}^{i}, u_{E}^{i}: \Delta_{E}(t) \rightarrow$ $\coprod_{E \in B_{t}} \Delta_{E}(i)^{m_{E}}$ the corresponding inclusions. Thus, we have the following commutative diagram

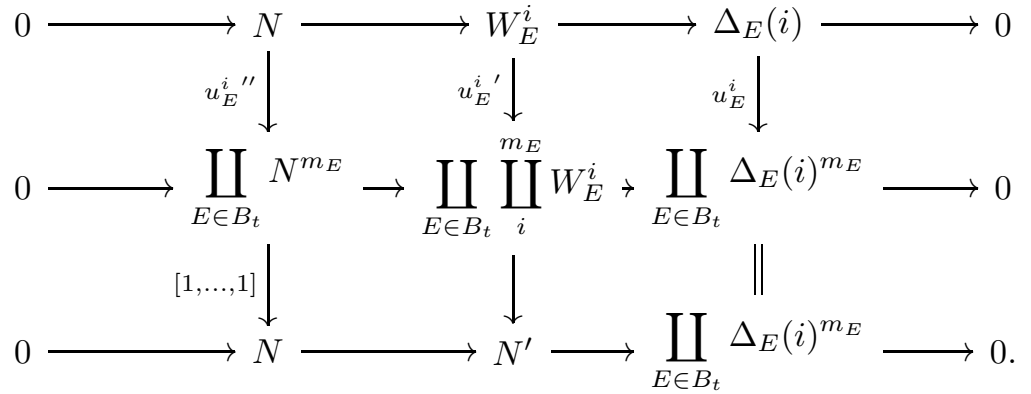

By Lemma 2.9, we have that $\operatorname{Ext}^{1}\left(\Delta(j), \coprod_{E \in B_{t}} \Delta_{E}(t)^{m_{E}}\right)=0$ for all $j \leq t$. In this way, after applying $\mathcal{C}(\Delta(j),-)$ to the exact sequence at the bottom, we have

$$
\begin{array}{r}
0 \rightarrow(\Delta(j), N) \rightarrow\left(\Delta(j), N^{\prime}\right) \rightarrow\left(\Delta(j), \coprod_{E \in B_{t}} \Delta_{E}(t)^{m_{E}}\right) \stackrel{\delta}{\rightarrow} \operatorname{Ext}^{1}(\Delta(j), N) \rightarrow \\
\operatorname{Ext}^{1}\left(\Delta(j), N^{\prime}\right) \rightarrow \operatorname{Ext}^{1}\left(\Delta(j), \coprod_{E \in B_{t}} \Delta_{E}(t)^{m_{E}}\right)=0
\end{array}
$$

Assume that $j<t$. Therefore, by hypothesis we have $\operatorname{Ext}^{1}(\Delta(j), N)=0$, and therefore $\operatorname{Ext}^{1}\left(\Delta(j), N^{\prime}\right)=0$. It only remains to verify the case $j=t$. Let $\Delta_{E^{\prime}}(t)=\mathcal{C}\left(-, E^{\prime}\right) / I_{\mathcal{B}_{t-1}}\left(-, E^{\prime}\right) \in \Delta(t)$. If $E^{\prime} \notin B_{t}$, we have $\operatorname{Ext}^{1}\left(\Delta_{E^{\prime}}(t), N\right)=0$; thus we have $\operatorname{Ext}^{1}\left(\Delta_{E^{\prime}}(t), N^{\prime}\right)=0$. If $E^{\prime} \in B_{t}$ the connection morphism $\delta$ is an epimorphism, and we conclude $\operatorname{Ext}^{1}\left(\Delta_{E^{\prime}}(t), N^{\prime}\right)=0$.

Lemma 3.6. Let $1 \leq t \leq n$. Let $N$ be a $\mathcal{C}$-module with $\operatorname{Ext}^{1}(\Delta(j), N)=0$ for all $j<t$. Then there exists an exact sequence $0 \rightarrow N \rightarrow Y \rightarrow X \rightarrow 0$ with $X \in \mathcal{F}(\{\Delta(1), \ldots, \Delta(t)\})$ and $Y \in \mathcal{Y}(\Delta)$.

Proof. By Lemma 3.5 we can construct exact sequences $0 \rightarrow N_{i+1} \rightarrow N_{i} \rightarrow Q_{i} \rightarrow 0$, $1 \leq i \leq t$, with $\operatorname{Ext}^{1}\left(\Delta(j), N_{i}\right)=0$ for all $j \leq i$, and $Q_{i}$ is a direct finite sum of objects from $\Delta(i)$. Set $N=N_{t+1}, N_{1}=Y$ and $X=Y / N$.

Thus, $Y \in \mathcal{Y}$ and there is a filtration for $X$

$$
0 \subset N_{t} / N \subset \cdots \subset N_{1} / N=Y / N=X
$$

such that $\left(N_{i+1} / N\right) /\left(N_{i} / N\right) \cong N_{i+1} / N_{i} \cong Q_{i}$.

Since $\Delta(1)$ consists only of projective objects, of particular interest is when $t=2$ in the above theorem. As a consequence, we have the following.

Lemma 3.7. For every $\mathcal{C}$-module $N$, there exists an exact sequence $0 \rightarrow N \rightarrow Y \rightarrow$ $X \rightarrow 0$ with $X \in \mathcal{F}(\Delta)$ and $Y \in \mathcal{Y}(\Delta)$.

The proof of Theorem 3.1 follows as in [ring2], and it follows from Lemma 3.3 . Furthermore, we may use duality in order also obtain left $\mathcal{F}(\Delta)$-approximations.

Since $\mathcal{C}$ is dualizing, we can define analogously as in Dlab and DR the category $\mathcal{F}(\nabla)$ as follows. Denote $\mathcal{F}\left(\Delta^{\circ}\right)$ as the full subcategory of covariant $\mathcal{C}$-modules with $\Delta^{\circ}$-filtration and by $\mathcal{F}(\nabla) \cong \mathcal{F}\left(\nabla^{\circ}\right)^{\circ}$ the category of all contravariant $\nabla$ filtered $\mathcal{C}$-modules. In this way we could try in the future to define the concept of characteristic category such as the category $\omega=\mathcal{F}(\Delta) \cap \mathcal{F}(\nabla)$ and to link the theorem obtained by Ringel (see Theorem 5 in [Rin2]) and Theorem 12 in [MVO2]. 


\section{EXAMPLES}

In this section, we exhibit some filtrations for the different Auslander-Reiten components to consider them as quasi-hereditary categories. We have already mentioned the importance of studying these components at the beginning of this work in relation to MVS3. Later we obtain a tilting category for $\mathbb{Z} A_{\infty}$ motivated by the theory developed in the third section. Finally, we show that tensor product of quasi-hereditary categories is again a quasi-hereditary category; in this way we can build more examples from others already given.

4.1. Auslander Reiten components seem like quasi-hereditary categories. Let $\Gamma=\left(\Gamma_{0}, \Gamma_{1}, \tau\right)$ be a translation quiver with translation $\tau: \Gamma_{0}^{\prime} \rightarrow \Gamma_{0}$ defined on a subset $\Gamma_{0}^{\prime} \subset \Gamma_{0}$. The vertices in $\Gamma_{0}$ which do not belong to $\Gamma_{0}^{\prime}$ are called projective. Given a translation quiver $\Gamma=\left(\Gamma_{0}, \Gamma_{1}, \tau\right)$, a semitranslation can be defined $\sigma: \Gamma_{1}^{\prime} \rightarrow \Gamma_{1}$, where $\Gamma_{1}^{\prime} \subset \Gamma_{1}$ is the set of all arrows $\alpha: a \rightarrow b$ with $b$ not projective, such that $\sigma(\alpha): \tau b \rightarrow a$ for $\alpha: a \rightarrow b$ Rin1, ASS. We can consider the mesh category $K(\Gamma, \sigma)$, i.e., the quotient category of the path category of $\left(\Gamma_{0}, \Gamma_{1}\right)$ modulo the mesh ideal. Remember that the mesh ideal in the path category of $\left(\Gamma_{0}, \Gamma_{1}\right)$ is the one generated by the elements $m_{x}=\Sigma_{\left\{\alpha \in \Gamma_{1}: t(\alpha)=x\right\}}$, where $t(\alpha)$ means the target of $\alpha$ and $x$ is a non-projective vertex.

In this part, we take $\Gamma$ as $\mathbb{Z} \Sigma$ where $\Sigma$ is one of the following: $D_{\infty}, A_{\infty}$ or $A_{\infty}^{\infty}$, or $\Gamma=\mathbb{N} \Sigma$, the full translation subquiver of $\mathbb{Z} \Sigma$, where $\Sigma$ is an extended Dynkin diagram. In this way, we will see that we think of the mesh category $K(\Gamma, \sigma)$ as a quasi-hereditary category; simultaneously, we can study the category of representations $\operatorname{Rep}(K \Gamma, \sigma)$ as the category of $K(\Gamma, \sigma)$-modules.

Now we show some of the filtrations for different mesh categories mentioned above. In order to do this, we pick out full subcategories $B_{i} \subset \mathcal{C}$, and after we consider the filtration $0=\mathcal{B}_{0} \subset \mathcal{B}_{1} \subset \cdots \cdots \subset \mathcal{B}_{i} \subset \cdots \mathcal{C}$, with $\mathcal{B}_{i}=\operatorname{add} B_{i}$ : the full subcategory of $\mathcal{C}$ consisting of directed summands of finite sums. Thus we obtain a filtration of $\mathcal{C}$ into closely additive subcategories.

The category $K\left(\mathbb{Z} A_{\infty}^{\infty}, \sigma\right)$. Let $B_{1}=\left\{E_{1}^{1}\right\}$, and $B_{i}=B_{i-1} \cup\left\{E_{j}^{i}\right\}_{1 \leq j \leq 4(i-1)}$ as is shown in the next picture.

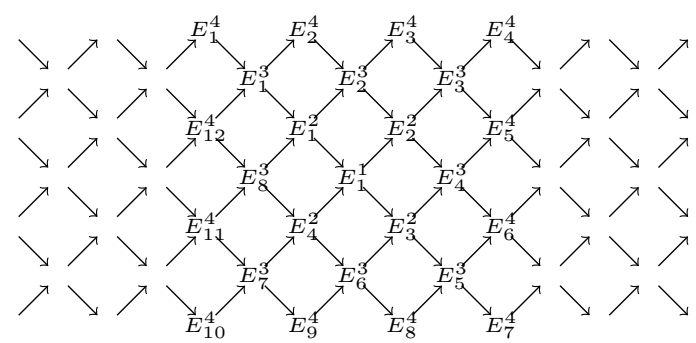

Labeled vertices in the graph $\mathbb{Z} \times A_{\infty}^{\infty}$.

Now we will see how the conditions of Theorem 2.7 are satisfied on $K\left(\mathbb{Z} A_{\infty}^{\infty}, \sigma\right)$. We only explain this case.

i) Indeed, by commutativity of the diagram, every path between a pair of non isomorphic indecomposable objects $E, E^{\prime} \in \mathcal{B}_{i}$ factors throughout an object $E^{\prime \prime} \in$ $\mathcal{B}_{i-1}$. Therefore, $\operatorname{rad}_{\mathcal{C}}\left(E, E^{\prime}\right)=I_{\mathcal{B}_{i-1}}\left(E, E^{\prime}\right)$.

ii) Let $X$ be an indecomposable object in $K\left(\mathbb{N} A_{\infty}^{\infty}, \sigma\right)$. If there is a path from $E_{1}$ to $X$, then $I_{\mathcal{B}_{1}}(-, X)=\mathcal{C}\left(-, E_{1}\right)$, else $I_{\mathcal{B}_{1}}(-, X)$ is the zero functor. This implies that $I_{\mathcal{B}_{1}}(-, X)$ is a projective $K\left(\mathbb{N} A_{\infty}^{\infty}, \sigma\right)$-module. For $i>1$, if $\left.\mathcal{C}(-, X)\right|_{\mathcal{B}_{i}}=0$, then there is no a path from $B$ to $X$ for any $B \in \mathcal{B}_{i}$, and $I_{\mathcal{B}_{i}}(-, X)=0$; if $X \in \mathcal{B}_{i}$ then $I_{\mathcal{B}_{1}}(-, X)=\mathcal{C}(-, X)$. Assume that $\left.\mathcal{C}(-, X)\right|_{\mathcal{B}_{i}} \neq 0$ and $X \notin \mathcal{B}_{i}$, 
then there is a finite set of indecomposable objects $\left\{E_{1}^{\prime}, \ldots, E_{r}^{\prime}\right\} \subset \mathcal{B}_{i}$ which are vertically aligned for which there is a path from $E_{j}^{\prime}$ to $X$ for $j=1, \ldots, r$. Thus, $I_{\mathcal{B}_{i}}(-, X)$ can be covered by $\coprod_{j=1}^{r} \mathcal{C}\left(-, E_{j}^{\prime}\right)$; therefore, there is an epimorphism $\mathcal{C}\left(-, E^{\prime}\right) \rightarrow I_{\mathcal{B}_{i}}(-, X) \rightarrow 0$ with $E^{\prime}=\coprod_{j=1}^{r} E_{j}^{\prime} \in \mathcal{B}_{i}$, which has a kernel that is a finite sum of copies of $\coprod \mathcal{C}\left(-, E^{\prime \prime}\right)$ with $E^{\prime \prime} \in \mathcal{B}_{i-1}$. Moreover, it can be covered by a projective module $\mathcal{C}\left(-, E_{i-1}\right), E_{i-1} \in \mathcal{B}_{i-1}$. As a result, we have an exact sequence

$$
\mathcal{C}\left(-, E_{i-1}^{\prime \prime}\right) \rightarrow \mathcal{C}\left(-, E_{i}^{\prime}\right) \rightarrow I_{\mathcal{B}_{i}}(-, X) \rightarrow 0
$$

Now, we illustrate the above situation for $I_{\mathcal{B}_{4}}(-, X)$ with $X=E_{7}^{5}$.

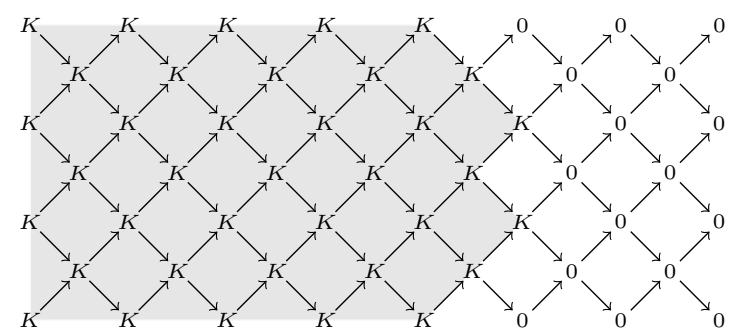

The functor $I_{\mathcal{B}_{4}}(-, X)$ with $X=E_{7}^{5}$.

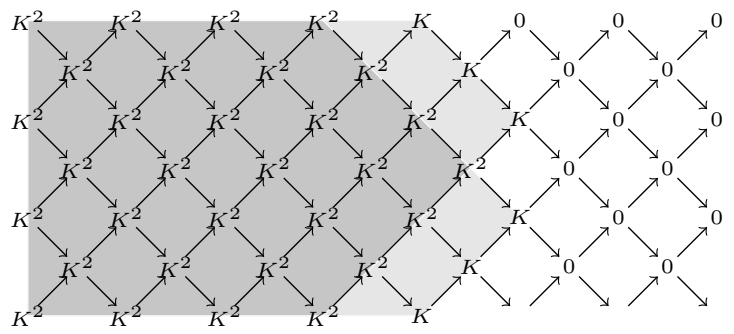

$I_{\mathcal{B}_{4}}(-, X)$ with $X=E_{7}^{5}$ can be covered by $\mathcal{C}\left(-, E_{5}^{4}\right) \coprod \mathcal{C}\left(-, E_{6}^{4}\right)$.

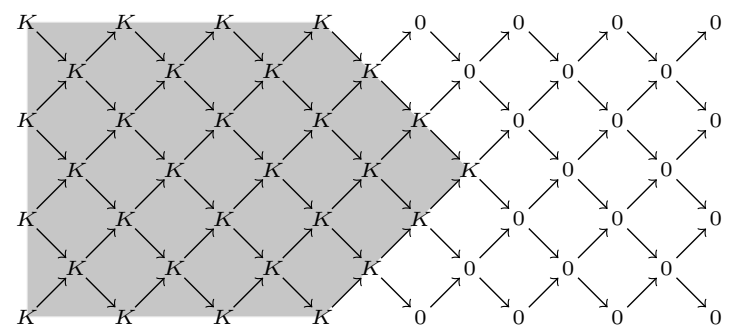

The kernel of $\mathcal{C}\left(-, E_{5}^{4} \coprod E_{6}^{4}\right) \rightarrow I_{\mathcal{B}_{4}}(-, X)$ is $\mathcal{C}\left(-, E_{4}^{3}\right)$.

In this way, we have an exact sequence $\mathcal{C}\left(-, E_{4}^{3}\right) \rightarrow \mathcal{C}\left(-, E_{5}^{4} \coprod E_{6}^{4}\right) \rightarrow I_{\mathcal{B}_{4}}(-, X) \rightarrow$ 0 with $E_{4}^{3} \in \mathcal{B}_{3}$ and $E_{5}^{4} \coprod E_{6}^{4} \in \mathcal{B}_{4}$.

The category $K\left(\mathbb{Z} A_{\infty}, \sigma\right)$. Let $B_{1}=\left\{E_{j}^{1}\right\}_{j \in \mathbb{Z}}$, and $B_{i}=B_{i-1} \cup\left\{E_{j}^{i}\right\}_{j \in \mathbb{Z}}$ as they appear in the next picture. 
THE AUSLANDER-REITEN COMPONENTS SEEN AS QUASI-HEREDITARY CATEGORIES27

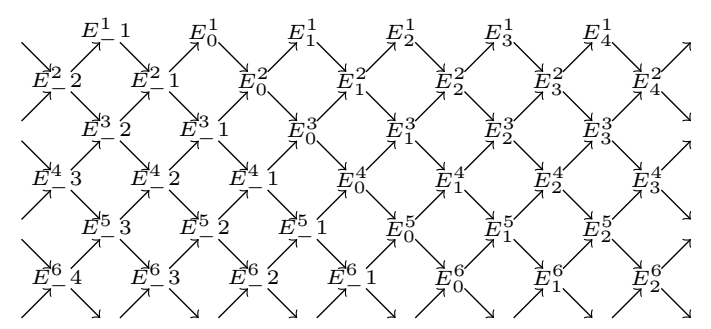

Labeled vertices in the graph $\mathbb{Z} \times A_{\infty}$

The category $K\left(\mathbb{Z} D_{\infty}, \sigma\right)$. Let $B_{1}=\left\{E_{1}^{1}\right\}$ and $B_{i}=\left\{\begin{array}{l}B_{i-1} \cup\left\{E_{j}^{i}\right\}_{1 \leq j \leq 2(i+1)}, \quad \text { if } i \text { is even; } \\ B_{i-1} \cup\left\{E_{j}^{i}\right\}_{1 \leq j \leq 2 i-1}, \quad \text { if } i \text { is odd. }\end{array}\right.$

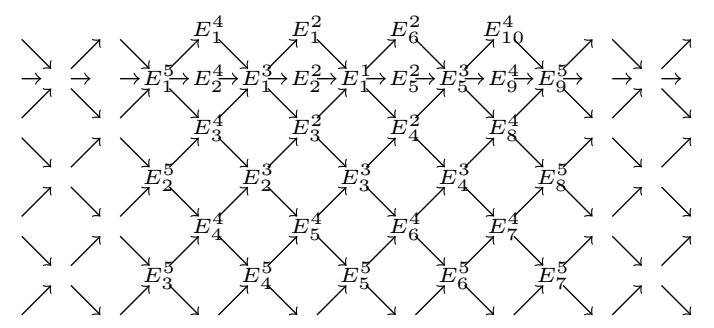

Labeled vertices in the graph $\mathbb{Z} \times D_{\infty}$

The categories $K\left(\mathbb{N} \tilde{D}_{m}, \sigma\right), K\left(\mathbb{N} \tilde{A}_{m}, \sigma\right), K\left(\mathbb{N} \tilde{E}_{6}, \sigma\right), K\left(\mathbb{N} \tilde{E}_{7}, \sigma\right)$ and $K\left(\mathbb{N} \tilde{E}_{8}, \sigma\right)$.

We start with $K\left(\mathbb{N} \tilde{D}_{m}, \sigma\right)$. First we label the vertices of $\tilde{D}_{n}$ as $\left(\tilde{D}_{n}\right)_{0}=$ $\{1,2, \ldots, n-1, n-1, n, n+1\}$. Let $A=\left\{x \in\left(\tilde{D}_{n}\right)_{0}: x\right.$ is a source vertex $\}$ and $B=\left\{x \in\left(\tilde{D}_{n}\right)_{0}: x\right.$ is a sink vertex $\}$. Now we label the indecomposable objects of $K\left(\mathbb{N} \tilde{D}_{m}, \sigma\right)$; first we label the vertices of $\{1\} \times \tilde{D}_{m}=\left\{E_{j}^{1}\right\}_{j \in A} \cup\left\{E_{j}^{2}\right\}_{j \in B}$. For $i \geq 3$, let $E_{j}^{i}=\tau^{-1}\left(E_{j}^{i-2}\right)$ and define $B_{1}=\left\{E_{j}^{1}\right\}_{j \in A}, B_{2}=B_{1} \cup\left\{E_{j}^{2}\right\}_{j \in B}$. For $i \geq 3$,

$$
B_{i}= \begin{cases}B_{i-1} \cup\left\{E_{j}^{i}\right\}_{j \in A}, & \text { if } i \text { is odd } \\ B_{i-1} \cup\left\{E_{j}^{i}\right\}_{j \in B}, & \text { if } i \text { is even }\end{cases}
$$

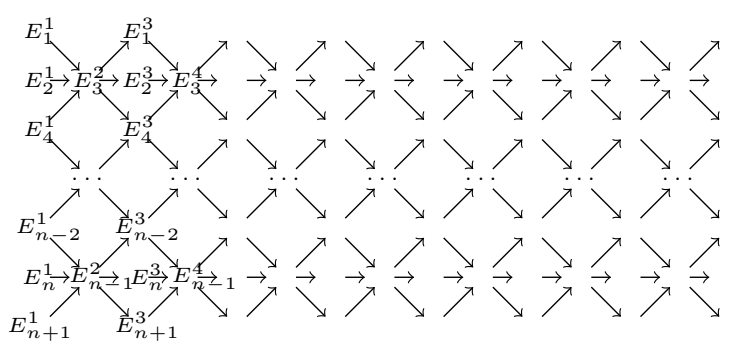

Labeled vertices in the graph $\mathbb{N} \times \tilde{D}_{m}$

Analogously, we can give a filtration for the rest of the categories: $K\left(\mathbb{N} \tilde{A}_{m}, \sigma\right)$, $K\left(\mathbb{N} \tilde{E}_{6}, \sigma\right), K\left(\mathbb{N} \tilde{E}_{7}, \sigma\right)$ and $K\left(\mathbb{N} \tilde{E}_{8}, \sigma\right)$ into subcategories. For example, for $K\left(\mathbb{N} \tilde{E}_{6}, \sigma\right)$, let $\left(\tilde{E}_{6}\right)_{0}=\{1,2, \ldots, 7\}$, and let $A=\{1,3,5,7\}, B=\{2,4,6\}$. Define $B_{1}=$ $\left\{E_{1}^{1}, E_{3}^{1}, E_{5}^{1}, E_{7}^{1}\right\}, B_{2}=B_{1} \cup\left\{E_{2}^{2}, E_{4}^{2}, E_{6}^{2}\right\}, B_{i}=B_{i-1} \cup\left\{E_{1}^{i}, E_{3}^{i}, E_{5}^{i}, E_{7}^{i}\right\}$, if $i$ is odd, and $B_{i}=B_{i-1} \cup\left\{E_{2}^{i}, E_{4}^{i}, E_{6}^{i}\right\}$, if $i$ is even. 


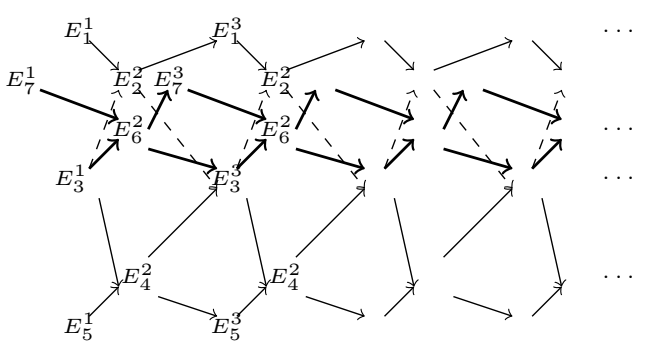

Labeled vertices in the graph $\mathbb{N} \times \tilde{E}_{6}$

4.2. A tilting subcategory in $\mathcal{C}=K\left(\mathbb{Z} A_{\infty}, \sigma\right)$. According with to filtration given above, although it is not finite, we could try to find tilting category $\mathcal{T} \subset$ $\operatorname{Mod}(\mathcal{C})$ in the category $\mathcal{F}(\Delta) \cap \mathcal{F}(\nabla)$ (see Rin2]). In this part, we found a tilting category in $\operatorname{Mod}(\mathcal{C})$ for this special case. First, we label the vertices of $\mathbb{Z} A_{\infty}$ as follows.

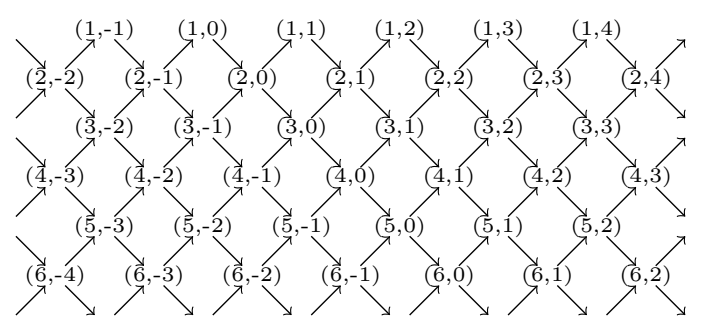

Labeled vertices in the graph $\mathbb{Z} \times A_{\infty}$

In this way, we can indentify the representations assigning a $K$-vector space $V_{i j}$ to each vertice $(i, j) \in \mathbb{N} \times \mathbb{Z}$.

Let $r \in \mathbb{Z}$ and consider the representation $T(r, 1)=\left\{V_{i j}\right\}$ defined as

$$
V_{i j}=\left\{\begin{array}{l}
K, \quad \text { if } i \geq r \text { and }-(i-r-1) \leq j \leq 1 \\
0 \quad \text { in other case, }
\end{array}\right.
$$

and a map between two adjacent $K$-vectorial spaces $V_{r s}$ and $V_{u v}$ is $1_{K}$ if $V_{r s}=$ $V_{u v}=K$ and 0 in other case.

In the same way, for any $s \in \mathbb{Z}$ we can define the moved representations of $T(r, 1)$ as follows. We define $T(r, s)=\left\{V_{i j}\right\}$ as

$$
V_{i j}=\left\{\begin{array}{l}
K, \quad \text { if } i \geq r \text { and }-(i-r-s) \leq j \leq s \\
0 \quad \text { in other case. }
\end{array}\right.
$$

Lemma 4.1. For all pairs $(r, s) \in \mathbb{N} \times \mathbb{Z}$ the representation $T(r, s)$ lies in $\mathcal{F}(\Delta)$.

Proof. Indeed for simplicity we only consider the representation $T(1,1)$. First, we observe that $T(1,1)^{(i)}=\mathcal{C}\left(-, E_{1}^{i}\right)$. On the other hand, let $X$ be an idecomposable object. By the mesh relations then each path in $I_{\mathcal{B}_{i-1}}\left(X, E_{1}^{i}\right)$ can be written in a unique way as $X \rightarrow E_{1}^{i-1} \stackrel{\alpha}{\rightarrow} E_{1}^{i}$ where $\alpha$ is the unique arrow from $E_{1}^{i-1}$ to $E_{1}^{i}$; therefore $I_{\mathcal{B}_{i-1}}\left(-, E_{1}^{i}\right) \cong \mathcal{C}\left(-, E_{1}^{i-1}\right)$. It follows that we have a chain

$$
0=T(1,1)^{(0)} \subset T(1,1)^{(1)} \subset T(1,1)^{(2)} \subset \cdots,
$$

such that $T(1,1)^{(i)} / T(1,1)^{(i-1)} \cong \mathcal{C}\left(-, E_{1}^{i}\right) / \mathcal{C}\left(-, E_{1}^{i-1}\right) \cong \mathcal{C}\left(-, E_{1}^{i}\right) / I_{\mathcal{B}_{i-1}}\left(-, E_{1}^{i}\right) \in$ $\Delta(i)$. Therefore $T(1,1) \in \mathcal{F}(\Delta)$.

Lemma 4.2. (i) $T(1, s)$ is a projective $\mathcal{C}$-module for all $s \in \mathbb{Z}$. 
(ii) For all $(r, s) \in \mathbb{N} \times \mathbb{Z}$, there is an exact sequence

$$
0 \rightarrow \mathcal{C}\left(-, E_{s}^{r-1}\right) \stackrel{j}{\rightarrow} T(1, s) \stackrel{p}{\rightarrow} T(r, s) \rightarrow 0 .
$$

(iii) Let $(r, s),\left(r^{\prime}, s^{\prime}\right) \in \mathbb{N} \times \mathbb{Z}$. Then $\operatorname{Hom}\left(\mathcal{C}\left(-, E_{s}^{r}\right), T\left(r^{\prime}, s^{\prime}\right)\right)=0$ if $s \neq s^{\prime}$ or $s=s^{\prime}$ and $r<r^{\prime}$.

(iv) For all $(r, s) \in \mathbb{N} \times \mathbb{Z}$, the projective $\mathcal{C}$-module $\mathcal{C}\left(-, E_{s}^{r}\right)$ has a resolution

$$
0 \rightarrow \mathcal{C}\left(-, E_{s}^{r}\right) \rightarrow T(1, s) \rightarrow T(r+1, s) \rightarrow 0 .
$$

Proof. To prove (i), consider the directed system of projective $\mathcal{C}$-modules: $\mathcal{C}\left(-, E_{s}^{1}\right) \subset$ $\mathcal{C}\left(-, E_{s}^{2}\right) \subset \cdots$. Clearly, it follows that $T(1, s)=\lim \mathcal{C}\left(-, E_{s}^{i}\right)$. Hence, $T(1, s)$ is a flat $\mathcal{C}$-module because it is a limite of projective $\mathcal{C}$-modules. Let be $0 \rightarrow A \rightarrow B \rightarrow$ $C \rightarrow 0$ an exact sequence of $\mathcal{C}$-modules. As a resultado, we have an exact sequence of $\mathcal{C}$-modules:

$$
0 \rightarrow T \otimes_{K} A \rightarrow T \otimes_{K} B \rightarrow T \otimes_{K} C \rightarrow 0
$$

If we denote $D=\operatorname{Hom}(-, K): L . F(\mathcal{C}) \rightarrow L . F\left(\mathcal{C}^{o p}\right), D(M)(X)=\operatorname{Hom}(M(X), K)$, the usual duality between the subcategories of locally finite $\mathcal{C}$-modules.

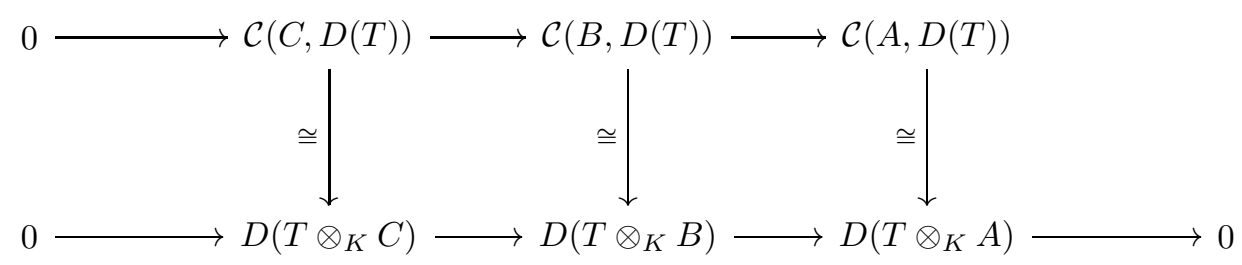

it follows that the exact sequence on the top is a short exact sequence. Thus, $D(T)$ is injective and therefore $T$ is projective. (ii) is clear, and it follows from the definition of $T(r, s)$. (iii) follows from the mesh relations in $\mathcal{C}$, finally (iv) follows straightforward from the definition.

Theorem 4.3. The full subcategory $\mathcal{T}$ of $\operatorname{Mod}(\mathcal{C})$ consisting of the family of $\mathcal{C}$ modules $\{T(r, s)\}_{(r, s) \in \mathbb{N} \times \mathbb{Z}}$ is a tilting category.

Proof. i) It is clear that pdim $T(r, s) \leq 1$ by (i) and (ii) in Lemma 4.2, ii) After applying $\operatorname{Hom}\left(-, T\left(r^{\prime}, s^{\prime}\right)\right)$ to the exact sequence (42), we obtain the following exact sequence:

$$
\begin{aligned}
& 0 \rightarrow \operatorname{Hom}\left(T(r, s), T\left(r^{\prime}, s^{\prime}\right)\right) \stackrel{p *}{\rightarrow} \operatorname{Hom}\left(T(1, s), T\left(r^{\prime}, s^{\prime}\right)\right) \stackrel{j *}{\rightarrow} \\
& \operatorname{Hom}\left(\mathcal{C}\left(-, E_{s}^{r-1}\right), T\left(r^{\prime}, s^{\prime}\right)\right) \stackrel{\partial}{\rightarrow} \operatorname{Ext}^{1}\left(T(r, s), T\left(r^{\prime}, s^{\prime}\right)\right) \rightarrow 0 .
\end{aligned}
$$

First, assume that $s \neq s^{\prime}$ or $s=s^{\prime}$ and $r-1<r^{\prime}$, then by Lemma 4.2, we have $\operatorname{Hom}\left(\mathcal{C}\left(-, E_{s}^{r-1}\right), T\left(r^{\prime}, s^{\prime}\right)\right)=0$ and $\operatorname{Ext}^{1}\left(T(r, s), T\left(r^{\prime}, s^{\prime}\right)\right)=0$. Assume that $s=s^{\prime}$ and $r-1 \geq r^{\prime}$, and let $h \in \operatorname{Hom}\left(\mathcal{C}\left(-, E_{s}^{r-1}\right), T\left(r^{\prime}, s^{\prime}\right)\right)$. If we write $h=\left\{h_{i j}: V_{i j} \rightarrow\right.$ $\left.V_{i j}^{\prime}\right\}$ as a map between representations, by commutativity, we can assume that each $h_{i j}=\lambda 1_{K}$ for some $\lambda \in K$ or zero. in this way, we have the following commutative diagram:

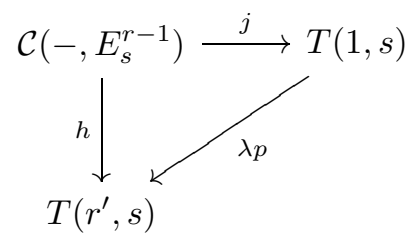

Therefore, $j *(\lambda p)=\lambda p j=h$. Hence, $j *$ is an epimorphism, and $\operatorname{Ext}^{1}\left(T(r, s), T\left(r^{\prime}, s^{\prime}\right)\right)=$ 0 .

iii) This condition follows directly from part (iv) of Lemma 4.2 , 
Finally, we illustrate the above theorem with an example

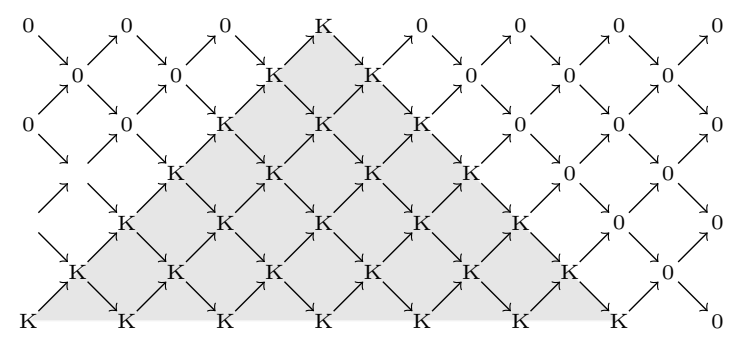

$T(1,1)$

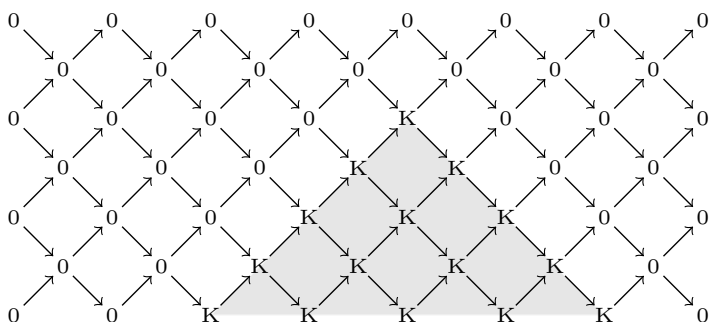

$T(3,1)$

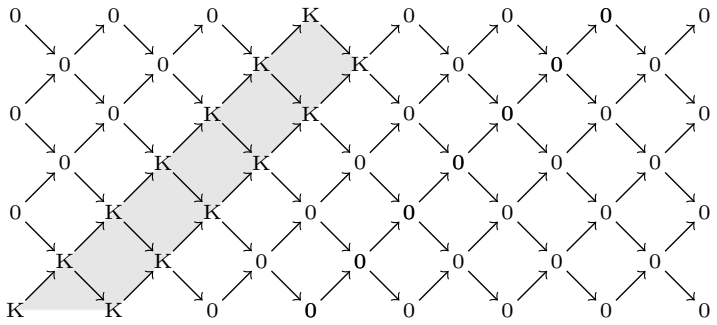

$$
\mathcal{C}\left(-, E_{1}^{2}\right)
$$

The above diagrams show how to obtain the exact sequence:

$$
0 \rightarrow \mathcal{C}\left(-, E_{1}^{2}\right) \rightarrow T(1,1) \rightarrow T(3,1) \rightarrow 0
$$

Of course many other filtrations for these categories can be given. For example, for the category $K\left(\mathbb{Z} A_{\infty}, \sigma\right)$, we can take $B_{1}=\left\{E_{1}^{1}\right\}$ and $B_{i}=B_{i-1} \cup\left\{E_{j}^{i}\right\}_{j \in \mathbb{N}}$ as shown in the next picture.

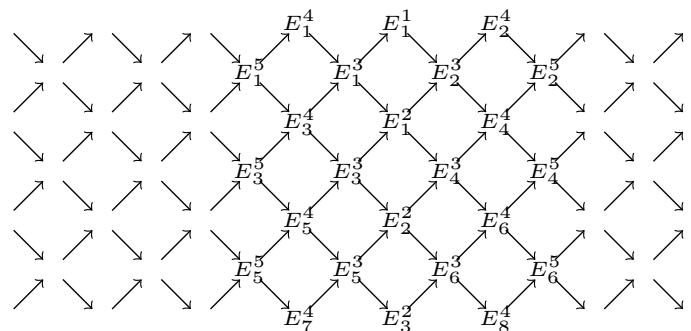

Labeled vertices in the graph $\mathbb{Z} \times A_{\infty}$

Another filtration for this category is given by $B_{1}=\left\{E_{1}^{1}\right\}$ and

$$
B_{i}=\left\{\begin{array}{l}
B_{i-1} \cup\left\{E_{j}^{i}\right\}_{1 \leq j \leq 2 i}, \quad \text { if } i \text { is even; } \\
B_{i-1} \cup\left\{E_{j}^{i}\right\}_{1 \leq j \leq 2 i-1}, \quad \text { if } i \text { is odd. }
\end{array}\right.
$$




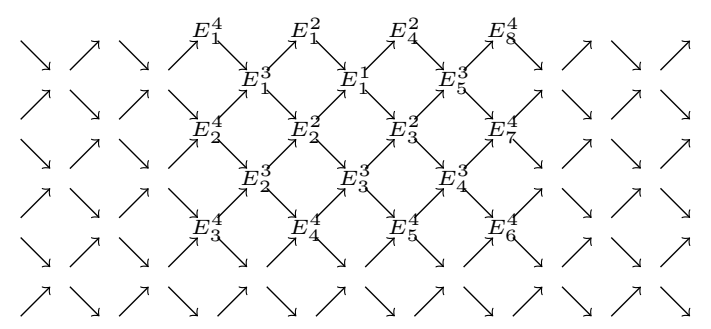

Labeled vertices in the graph $\mathbb{Z} \times A_{\infty}$

Observe that filtrations of the same style for the above examples could be given. Therefore, a category $\mathcal{C}$ can be considered cuasi-hereditary with respect to different filtrations.

4.3. Tensor product of quasi-hereditary categories. Let $K$ be a field. Assume that $\mathcal{C}_{1}$ and $\mathcal{C}_{2}$ are quasi-hereditary $K$-categories. In this section, we show that the tensor product $\mathcal{C}_{1} \otimes_{K} \mathcal{C}_{2}$ is quasi-hereditary.

First we remember some facts about the tensor product $\mathcal{C}_{1} \otimes \mathcal{C}_{2}$ of two categories (see $[\mathrm{M}]$ ). This is the category whose class of objects is $\left|\mathcal{C}_{1}\right| \times\left|\mathcal{C}_{2}\right|$, where the abelian group of morphisms from $\left(X_{1}, X_{2}\right)$ to $\left(Y_{1}, Y_{2}\right)$ is the ordinary tensor product of $K$ vectorial spaces $\mathcal{C}\left(X_{1}, Y_{1}\right) \otimes_{K} \mathcal{C}\left(X_{1}, Y_{1}\right)$

Consider additive functors $F: \mathcal{C}_{1} \rightarrow \operatorname{Mod}(K), G: \mathcal{C}_{2} \rightarrow \operatorname{Mod}(K)$. They induce the additive bifunctor

$$
F \otimes G: \mathcal{C}_{1} \otimes \mathcal{C}_{2} \rightarrow \operatorname{Mod}(K)
$$

wich is defined by $F \otimes G((X, Y))=F(X) \otimes G(Y)$. Assume that $I(-, ?)$ and $J(-, ?)$ are two-sided ideals in $\mathcal{C}_{1}$ and $\mathcal{C}_{2}$ respectively. We can then see that $J(-, ?) \otimes \mathcal{C}_{2}(-, ?)$ is a two-sided ideal in $\mathcal{C}_{1} \otimes \mathcal{C}_{2}$.

Theorem 4.4. Let $\mathcal{C}_{1}$ and $\mathcal{C}_{2}$ be quasi-hereditary $K$-categories. Then the tensor product $\mathcal{C}_{1} \otimes \mathcal{C}_{2}$ is quasi-hereditary.

Proof. Let

$$
\begin{aligned}
& \mathcal{C}_{1}(-, ?) \supset I_{1}(-, ?) \supset I_{2}(-, ?) \supset I_{3}(-, ?) \supset \cdots \\
& \mathcal{C}_{2}(-, ?) \supset J_{1}(-, ?) \supset J_{2}(-, ?) \supset J_{3}(-, ?) \supset \cdots
\end{aligned}
$$

the corresponding heredity chains of ideals.

We can then can form a chain of ideals:

$$
\begin{gathered}
\mathcal{C}_{1}(-, ?) \otimes \mathcal{C}_{2}(-, ?) \supset \mathcal{C}_{1}(-, ?) \otimes J_{1}+I_{1} \otimes \mathcal{C}_{2}(-, ?) \supset \mathcal{C}_{1}(-, ?) \otimes J_{2}+I_{1} \otimes \mathcal{C}_{2}(-, ?) \supset \\
\mathcal{C}_{1}(-, ?) \otimes J_{2}+I_{2} \otimes \mathcal{C}_{2}(-, ?) \supset \mathcal{C}_{1}(-, ?) \otimes J_{3}+I_{2} \otimes \mathcal{C}_{2}(-, ?) \supset \cdots
\end{gathered}
$$

We show that the above is a heredity chain.

Consider the inclusion

$$
\frac{\mathcal{C}_{1}(-, ?) \otimes \mathcal{C}_{2}(-, ?)}{\mathcal{C}_{1}(-, ?) \otimes J_{2}+I_{1} \otimes \mathcal{C}_{2}(-, ?)} \supset \frac{\mathcal{C}_{1}(-, ?) \otimes J_{1}+I_{1} \otimes \mathcal{C}_{2}(-, ?)}{\mathcal{C}_{1}(-, ?) \otimes J_{2}+I_{1} \otimes \mathcal{C}_{2}(-, ?)}
$$


Observe that

$$
\begin{aligned}
\frac{\mathcal{C}_{1}(-, ?) \otimes \mathcal{C}_{2}(-, ?)}{\mathcal{C}_{1}(-, ?) \otimes J_{2}+I_{1} \otimes \mathcal{C}_{2}(-, ?)} & \cong \frac{\frac{\mathcal{C}_{1}(-, ?) \otimes \mathcal{C}_{2}(-, ?)+I_{1} \otimes \mathcal{C}_{2}(-, ?)}{I_{1} \otimes \mathcal{C}_{2}(-, ?)}}{\frac{\mathcal{C}_{1}(-, ?) \otimes J_{2}+I_{1} \otimes \mathcal{C}_{2}(-, ?)}{I_{1} \otimes \mathcal{C}_{2}(-, ?)}} \\
& \cong \frac{\frac{\mathcal{C}_{1}(-, ?) \otimes \mathcal{C}_{2}(-, ?)}{I_{1} \otimes \mathcal{C}_{2}(-, ?)}}{\frac{\mathcal{C}_{1}(-, ?) \otimes J_{2}}{I_{1} \otimes J_{2}}} \\
& \cong \frac{\frac{\mathcal{C}_{1}(-, ?)}{I_{1}} \otimes \mathcal{C}_{2}(-, ?)}{\frac{\mathcal{C}_{1}(-, ?)}{I_{1}} \otimes J_{2}} \\
& \cong \frac{\mathcal{C}_{1}(-, ?)}{I_{1}} \otimes \frac{\mathcal{C}_{2}(-, ?)}{J_{2}}
\end{aligned}
$$

On the other hand,

$$
\begin{aligned}
\frac{\mathcal{C}_{1}(-, ?) \otimes J_{1}+I_{1} \otimes \mathcal{C}_{2}(-, ?)}{\mathcal{C}_{1}(-, ?) \otimes J_{2}+I_{1} \otimes \mathcal{C}_{2}(-, ?)} & \cong \frac{\frac{\mathcal{C}_{1}(-, ?) \otimes J_{1}+I_{1} \otimes \mathcal{C}_{2}(-, ?)}{I_{1} \otimes J_{1}}}{\frac{\mathcal{C}_{1}(-, ?) \otimes J_{2}+I_{1} \otimes \mathcal{C}_{2}(-, ?)}{I_{1} \otimes J_{1}}} \\
& \cong \frac{\frac{\mathcal{C}_{1}(-, ?) \otimes J_{1}}{I_{1} \otimes J_{1}}}{\frac{\mathcal{C}_{1}(-, ?) \otimes J_{2}}{I_{1} \otimes J_{2}}} \\
& \cong \frac{\frac{\mathcal{C}_{1}(-, ?)}{I_{1}} \otimes J_{1}}{\frac{\mathcal{C}_{1}(-, ?)}{I_{1}} \otimes J_{2}} \\
& \cong \frac{\mathcal{C}_{1}(-, ?)}{I_{1}} \otimes \frac{J_{1}}{J_{2}}
\end{aligned}
$$

In this manner proving that $\frac{\mathcal{C}_{1}(-, ?)}{I_{1}} \otimes \frac{J_{1}}{J_{2}}$ is a heredity ideal of $\frac{\mathcal{C}_{1}(-, ?)}{I_{1}} \otimes \frac{\mathcal{C}_{2}(-, ?)}{J_{2}}$ is sufficient.

(i) It is clear that $\left[\mathcal{C}_{1}(-, ?)\right]^{2}=\mathcal{C}_{1}(-, ?)$. This implies that

$$
\left[\frac{\mathcal{C}_{1}(-, ?)}{I_{1}}\right]^{2}=\frac{\mathcal{C}_{1}(-, ?)^{2}+I_{1}}{I_{1}}=\frac{\mathcal{C}_{1}(-, ?)}{I_{1}}
$$

Thus, we have

$$
\left[\frac{\mathcal{C}_{1}(-, ?)}{I_{1}} \otimes \frac{J_{1}}{J_{2}}\right]^{2}=\left[\frac{\mathcal{C}_{1}(-, ?)}{I_{1}}\right]^{2} \otimes\left[\frac{J_{1}}{J_{2}}\right]^{2}=\frac{\mathcal{C}_{1}(-, ?)}{I_{1}} \otimes \frac{J_{1}}{J_{2}}
$$

It follows that $\frac{\mathcal{C}_{1}(-, ?)}{I_{1}} \otimes \frac{J_{1}}{J_{2}}$ is idempotent.

(ii) Observe that $\operatorname{rad}\left[\frac{\mathcal{C}_{1}(-, ?)}{I_{1}} \otimes \frac{\mathcal{C}_{2}(-, ?)}{J_{2}}\right]=\operatorname{rad}\left[\frac{\mathcal{C}_{1}(-, ?)}{I_{1}}\right] \otimes \frac{\mathcal{C}_{2}(-, ?)}{J_{2}}+\frac{\mathcal{C}_{1}(-, ?)}{I_{1}} \otimes \operatorname{rad}\left[\frac{\mathcal{C}_{2}(-, ?)}{J_{2}}\right]$.

Since $\mathcal{C}_{1}$ and $\mathcal{C}_{2}$ are quasi-hereditary, we have

It follows that

$$
\frac{\mathcal{C}_{1}(-, ?)}{I_{1}} \operatorname{rad}\left[\frac{\mathcal{C}_{1}(-, ?)}{I_{1}}\right] \frac{\mathcal{C}_{1}(-, ?)}{I_{1}}=\frac{J_{1}}{J_{2}} \operatorname{rad}\left[\frac{\mathcal{C}_{2}(-, ?)}{J_{2}}\right] \frac{J_{1}}{J_{2}}=0 .
$$

$$
\begin{array}{r}
\left(\frac{\mathcal{C}_{1}(-, ?)}{I_{1}} \otimes \frac{J_{1}}{J_{2}}\right) \operatorname{rad}\left[\frac{\mathcal{C}_{1}(-, ?)}{I_{1}} \otimes \frac{\mathcal{C}_{2}(-, ?)}{J_{2}}\right]\left(\frac{\mathcal{C}_{1}(-, ?)}{I_{1}} \otimes \frac{J_{1}}{J_{2}}\right) \cong \\
\frac{\mathcal{C}_{1}(-, ?)}{I_{1}} \operatorname{rad}\left[\frac{\mathcal{C}_{1}(-, ?)}{I_{1}}\right] \frac{\mathcal{C}_{1}(-, ?)}{I_{1}} \otimes \frac{J_{1}}{J_{2}} \frac{\mathcal{C}_{2}(-, ?)}{J_{2}} \frac{J_{1}}{J_{2}}+\left(\frac{\mathcal{C}_{1}(-, ?)}{I_{1}}\right)^{3} \otimes \frac{J_{1}}{J_{2}} \operatorname{rad}\left[\frac{\mathcal{C}_{2}(-, ?)}{J_{2}}\right] \frac{J_{1}}{J_{2}}
\end{array}
$$

This is,

$$
\left(\frac{\mathcal{C}_{1}(-, ?)}{I_{1}} \otimes \frac{J_{1}}{J_{2}}\right) \operatorname{rad}\left[\frac{\mathcal{C}_{1}(-, ?)}{I_{1}} \otimes \frac{\mathcal{C}_{2}(-, ?)}{J_{2}}\right]\left(\frac{\mathcal{C}_{1}(-, ?)}{I_{1}} \otimes \frac{J_{1}}{J_{2}}\right) \cong 0
$$


(iii) Observe that $\frac{J_{1}(-, ?)}{J_{2}(-, ?)}$ is a projective $\frac{\mathcal{C}_{2}}{J_{2}}$-module. Thus, $\frac{\mathcal{C}_{1}(-, ?)}{I_{1}} \otimes \frac{J_{1}(-, ?)}{J_{2}(-, ?)}$ is a projective $\frac{\mathcal{C}_{1}}{J_{1}} \otimes \frac{\mathcal{C}_{2}}{J_{2}}$-module.

\section{ACKNOWLEDGEMENTS}

The author would like to thank Prof. Roberto Martínez-Villa for introducing him to the study of the quasi-hereditary algebras and for his valuable comments and suggestions for the preparation of this work. The author would also like to thank PROMEP/103.5/13/6535 for the financial support received for the development of this project.

\section{REFERENCES}

[AF] Anderson F. W., Fuller K. R., Rings and Categories of Modules. Graduate Texts in Mathematics 13, Springer- Verlag, 1974.

[ASS] Assem I., Simson D. and Skowroński A., Elements of the representation theory of associative algebras 1: Techniques of representation theory, London Mathematical Society Student Texts, vol. 65, Cambridge Univ. Press, Cambridge, 2006.

[A] Auslander M., Representation Theory of Artin Algebras I, Communications In Algebra, 1(3), 177-268 (1974).

[A2] Auslander M., Applications of morphisms determined by modules. Representation theory of algebras (Proc. Conf., Temple Univ., Philadelphia, Pa.,1976), pp. 245-327. Lecture Notes in Pure Appl. Math., Vol. 37, Dekker, New York, 1978.

[A3] Auslander M., Maurice Auslander, A Functorial Approach to Representation Theory, Representations of Algebras, Lecture Notes in Mathematics, vol. 944, Springer Verlag, 1982, pp. 105-179.

[AB] Auslander, M., Buchweitz, R. The homological theory of maximal Cohen-Macaulay approximations. Colloque en l'honneur de Pierre Samuel (Orsay 1987). M'em. Soc. Math. France (N.S.) 38, 5-37 (1989)

[AQM] Auslander M., Representation dimension of Artin Algebras I. Queen Mary College Mathematics Notes, 1971., Selected Works of Maurice Auslander, Part 1 AMS, 505574 .

[APR] Auslander M., Platzek M. I., and Reiten I., Coxeter functors without diagrams. Trans. Amer. Math. Soc. 250 (1979), 1-46.

[AuRe] Auslander, M., Reiten, I. Applications of contravariantly finite subcategories. Adv.Math. 86(1), 111-151 (1991).

[AR] Auslander M., and Reiten I., Stable Equivalence of Dualizing $R$-Varietes Adavances in Mathematics Vol.12, No.3, (1974), 306-366.

[AR2] Auslander M., and Reiten I. Stable equivalence of Artin algebras. Proceedings of the Conference on Orders, Group Rings and Related Topics (Ohio State Univ., Columbus, Ohio, 1972), pp. 8-71. Lecture Notes in Math., Vol. 353, Springer, Berlin, 1973.

[ARS] Auslander M., Reiten I., and Smalø S. O., Representation Theory of Artin Algebras. Cambridge University Press. 1995.

[BR] Bautista R., Irreducible morphisms and the radical of a category. An. Inst. Mat. Univ. Nac. Autónoma México 22 (1982), 83-135 (1983).

[Scott] Cline E., Parshall B., and Scott L., Simulating algebraic geometry with algebra, I: The algebraic theory of derived categories, Proc. Sympos. Pure Math., 47 (1987), pp. 271-281 Part 2.

[CPS1] Cline E., Parshall B., and Scott L., Algebraic stratification in representation categories, Journal of Algebra 117, Issue 2, 504-521304 (1988).

[CPS2] Cline E., Parshall B., and Scott L., Derived categories, quasi-hereditary algebras and algebraic groups, in: Proceedings of the Ottawa-Moosonee Workshop in Algebra, 1987, Mathematics Lecture Notes series, Carleton University and Université d'Ottawa, 1988.

[Dlab] Dlab, V., Quasi-hereditary algebras, Appendix to Drozd, Yu.A., Kirichenko, V.V., Finite dimensional algebras. Springer-Verlag (1993.)

[DR] Dlab V., Ringel C. M., Quasi-hereditary algebras. Illinois J. Math. 33 (1989), no. 2, 280-291.

[MV] Martínez-Villa R., Applications of Koszul algebras: The preprojective algebra, Representation Theory of Algebras, CMS Conf. Proc. vol. 18, Amer. Math. Soc., Providence, RI (1996), pp. 487-504.

[MVO1] Martínez-Villa R., Ortiz-Morales M., Tilting Theory and Functor Categories I. Classical Tilting, Appl. Categ. Structures, doi: 10.1007/s10485-013-9322-y. 
[MVO2] Martínez-Villa R., Ortiz-Morales M., Tilting Theory and Functor Categories II. Generalized Tilting, Appl. Categ. Structures, doi: 10.1007/s10485-011-9266-z.

[MVO3] Martínez-Villa R., Ortiz-Morales M., Tilting Theory and Functor Categories III. The Maps Category, Int. J. Algebra 5 (2011), no. 11, 529-561.

[MVS1] Martínez-Villa R., Solberg Ø., Graded and Koszul categories, Appl. Categ. Structures, doi:10.1007/s10485-009-9191-6, in press.

[MVS2] Martínez-Villa R., Solberg Ø., Artin and Schelter regular algebras and categories, Journal of Pure and Applied Algebra, in press.

[MVS3] Martínez-Villa R., Solberg, Ø., Noetherianity and Gelfand-Kirillov dimension of components. J. Algebra 323 (2010), no. 5, 1369-1407.

[M] Mitchell, B., Rings with several objects. Advances in Math. 8, 1-161 (1972).

[P] Popescu N., Abelian categories with applications to rings and modules. Acad. Press (1973).

[ReVan] Reiten I., Van Den Bergh M., Noetherian Hereditary Abelian Categories Satisfying Serre Duality. Journal of the American Mathematical Society, Vol. 15, No. 2, 295-366 (2002).

[Rin1] Ringel C. M., Tame algebras and integral quadratic forms, Lecture Notes in Mathematics, 1099, Springer (1984).

[Rin2] Ringel C. M., The category of modules with good filtrations over a quasi-hereditary algebra has almost split sequences, Math. Z.208, 209-223.

[S] Stenström Bo., Rings of Quotients, 217, Springer-Verlang, New York 1975.

[SM] Sounders Mac Lane. Categories for the Working Mathematician. Springer-Verlag, New York, I971.

Facultad de Ciencias, Universidad Autónoma del Estado de México, México.

E-mail address: mortizmo@uaemex.mx

$U R L:$ http://www . uaemex.mx/fciencias/ 